M. H.! Zum Schlusse ist noch die Frage der Bean sta ndung sgrenze zu erörtern. Bei sämtlichen Würsten, die unter Wasserzusatz hergestellt werden, ist, mit Ausnahme der frischen Bratwurst, die Bereitung, wie die zahlreichen mitgeteilten Beobachtungen klar erwiesen haben, mit einem meist sehr beträchtlichen Wasserverlust verbunden, sodaß die Beanstandungsgrenze bei den Wurstersatzsorten, bei welchen erhebliche Schüttungen in Frage kommen, wie bei 'Brühwürsten, Leberwürsten, Schwartemagen, ganz unbedenklich tiefer festgesetzt werden kann, als der zugelassene Wasserzusatz. Nach meinem Dafürhalten wird dies zweckmäßig durch den Satz zum Ausdruck gebracht, daß Würste, welche einen Wasserüberschuß, berechnet aus der Differenz: Gesamt-Wassergehalt minus organisches Nichtfett $X 4$, ron mehr als 15 Teilen auf 100 Teile ungewässerte Wurstmasse aufweisen, nicht mehr als so hergestellt gelten können, wie der Verbraucher berechtigterweise erwarten darf.

Den verschiedentlich gemachten Vorschlag, die der Berechnung des MindestWassergehaltes zugrunde gelegte Verhältniszahl 4 durch eine die Berechnung des wahrscheintichen Fremdwassergehaltes ermöglichende Verhältniszahl etwa von $3,5 \mathrm{zu}$ ersetzen, möchte ich nicht unterstützen. Schon vom Standpunkte der Billigkeit aus dürfte es vorzuziehen sein, an der eine größere Sicherheit der Beurteilung verbürgenden Zahl 4 festzuhalten. Wohl bin ich mir bewußt, daß nach meinen Vorschlägen manche übermäßaige Wasserzusätze der Feststellung noch entgehen. Jedoch muß es, da der wirkliche Fremdwassergehalt doch nicht in allen Fällen annähernd genau ermittelt werden kann, genügen, sagen zu können, daß mindestens so oder soviel Wasser zugesetzt wurde. Der Hauptzweck solcher für die Praxis bestimmten Methoden ist doch durch den erzieherischen Wert gekennzeichnet, welcher darin liegt, daß die Produzenten durch die Ermöglichung der Aufdeckung von Mißständen in der Herstellung von Lebensmitteln zur Abstellung derselben angehalten werden. Diesem $Z$ week aber dürfte die Annahme der von mir empfohlenen Bearstandungsgrenzen in genügender Weise gerecht werden.

Dr. Seel (zur Geschäftsordnung): Um die Diskussion abzukürzen, möchte ich den Vorschlag machen, daß ich zuerst noch meinen Vortrag halte und daß dann die Diskussion eröffnet wird.

Der Vorsitzende: Es steht noch aus der Vortrag des Herrn Seel: Die Beurteilung von Wurstwaren auf Grund der chemischen und mikroskopischen Untersuchungen.

Ich glaube, der eben gemachte Vorschlag ist nicht unpraktisch, ich bitte Herrm Seel zunächst seinen Vortrag zu halten.

\title{
Die Beurteilung von Wurstwaren auf Grund der chemischen und mikroskopischen Untersuchung.
}

Von

Eugen Seel-Stuttgart.

Wie ich schon an anderer Stelle ${ }^{1}$ ) ausgeführt habe, gehört die Untersuchung und Beurteilung von Wurstwaren zu den schwierigsten Aufgaben der Nahrungsmittel-

1) Zeitschr. f. Fleisch- und Milchhygiene 1918, 28, 312 und 325. 
kontrolle, wenn diese in gewissenhafter Weise vorgenommen wird und sich nicht auf die Ausführung der Geschmacksprobe oder eine oberflächliche Wurstbeschau oder eine abgekürzte Untersuchung beschränkt.

$\mathrm{Zu}$ einer richtigen Beurteilung, wie sie besonders für gerichtliche Fälle wegen der hier entstehenden Folgen notwendig ist, kann man aber nur auf Grund einer gründlichen Untersuchung der Zusammensetzung der betreffenden Wurst gelangen. Hierzu ist eine a u f $\ddot{\mathrm{h} h \mathrm{rli}} \mathrm{c}$ h e chemische und mikroskopische Untersuchung unerläßlich, denn die Wurstherstellung ist leider in den meisten Fällen immer noch "Vertrauenssache“, die Wurst selbst ein „Kunstprodukt" und meist eine zum sofortigen Genusse „fertige Speise“.

Der Sachverständige muß nicht nur mit den örtlichen Verbältnissen, den orts* üblichen Gebräuchen und nicht zuletzt mit den Geheimnissen des Wurstkessels überhaupt vertraut, sondern auch imstande sein, die einzelnen Bestandteile der Wurst zu erkennen, mit anderen Worten, mit Hilfe der chemischen und mikroskopischen Untersuchung die Zusammensetzung der Wurst zu erforschen und so dem Geheimnis der Wurstbereitung auf den Grund zu gehen. Hierzu sind chemische und mikroskopischhistologische Kenntnisse notwendig. Der Chemiker muB sich daher die letzteren auch aneignen. Daß dies möglich ist, habe ich gemeinsam mit Herrn Dr. Reihling und Fräulein $\mathrm{Zeeb^{1 }}$ ) in einer bereits in Druck gegebenen Abhandlung zu zeigen begonnen.

Diese mikroskopisch-histologische Untersuchung bildet die notwendige Ergänzung der chemischen Analyse. Wie letztere zweckmäßig ausgeführt wird, habe ich $^{2}$ ) in früheren Arbeiten, namentlich aber gemeinsam mit Herrn Dr. Reihling in einer in Arbeit befindlichen längeren Abhandlung angegeben, sodaß ich mich heute auf die Beurteilung der Wurstwaren auf Grund der chemischen und mikroskopischen Untersuchung beschränken kann.

Auf chemischem Wege wurde bisher meistens nur die allgemein e Zusammensetzung der Wurst, und zwar deren Gehalt an Wasser, Fett, Stickstoffsubstanzen und Salzen ermittelt. Will man nun z. B. nur den ersteren, d. h. den Wassergehalt beurteilen, so muß man dennoch wissen, welche Arten von Fett und besonders von Stickstoffsubstanzen und in welcher annähernden Menge diese vorhanden sind. Hier muß schon die mikroskopische Untersuchung helfend eingreifen, weil die Stickstoffsubstanzen der meist gekochten Würste durch chemische Verfahren nur mangelhaft getrennt werden können, der Wassergehalt der Grundsubstanzen, d. h. der das Eiweiß enthaltenden Gewebe und Organe aber sehr versehieden ist. So z. B. enthält nach A bderhalden ${ }^{3}$ ) das elastische Bindegewebe, das in Lungen, derben Sehnen, Knorpeln usw. den Hauptbestandteil bildet, 50\% Wasser, während das leimgebende Bindegewebe etwa $80 \%$ Wasser, das Muskeleiweiß etwa $75 \%$ Wasser enthält. Es muß daher auf mikroskopischem Wege festgestellt werden, welche Grundsubstanzen und somit welche Eiweißarten und in welcher Menge dieselben vorhanden sind. Einzelne dieser Stickstoffsubstanzen rermögen noch dazu bei der Wurstbereitung mehr Wasser zu binden, als sie im ursprünglichen Zustande enthalten. Es kann je ein Teil Gelatine,

1) Diese Zeitschrift $1919, \mathbf{3 7}, 1$.

2) Diese Zeitschrift 1916, 32, 13 und 1918, 35, 393.

3) A bderhalden, Lehrbuch der physiologischen Chemie in Vorlesungen 1915, S. 800; siehe auch Diese Zeitschrift 1918, 35, 397. 
die aus leimgebenden Geweben entsteht und ein durchschnittlicher Bestandteil des Bindegewebes ist, mit 100 Teilen Wasser noch eine Gallerte bilden, also noch fest. werden.

Auch aus anderen Gründen ist die mikroskopische Untersuchung unerläblich, z. B. um die einzelnen Bestandteile der Würste zu erkennen, wie die wertvolle Leber in Leberwürsten oder wie geringwertige, unappetitliche und gar verbotene Organe (Lunge, Blättermagen, Gekröse, Hoden, Augen usw.) in Würsten versehiedener Art oder Bezeichnung. Ebenso ist die Prüfung auf gesundheitsschädliche Organe, hauptsãchlich auf mikroskopischem Wege vorzunehmen, um Anhaltspunkte für diesbezügliche Beurteilung zu erhalten, z. B. bei Wurstvergiftungen, denn einzelne Organe gehen leicht in Fäulnis über oder sind Träger von Fäulniserregern.

Auch für die richtige Berechnung des Nährwerts einer Wurst ist die Erkennung der einzelnen Eiweißarten notwendig; hierzu ist die Feststellung der Verdaulichkeit, die auf chemischem Wege einigermaßen ermittelt wird, unerläblich; denn nur auf Grund dieser Eigenschaft läßt sich der wahre Wert einer Wurst beurteilen. Es kann z. B. eine Wurst hauptsächlich aus minderwertigen, größtenteils unverdaulichen Stickstoffsubstanzen, wie Knorpeln, Sehnen u. dergl. bestehen und doch hinsichtlich ihres Gehaltes an Wasser, Stickstoffsubstanz, Fett und Salzen analysenfest hergestellt sein und ein bestimmtes Verhältnis von Wasser zu organischem Nichtfett bei der Untersuchung aufweisen. Andererseits kann eine aus leichtverdaulichen und guten Stickstoffsubstanzen, wie Muskelfaser, Leber, Blut $\mathbf{u}$. dergl. unter Zugabe von etwas Sulze bereitete Wurst eine gewisse Verhältniszahl überschreiten und daher wegen eines angeblich zu hohen Wassergehaltes beanstandet werden, trotzdem sie preiswerter und nahrhafter ist als erstere. Man übersiebt dabei, daß in dem übermäBigen Zusatz minderwertiger Fleischteile eine größere Verfälsehung nach \& 10 des Nahrungsmittelgesetzes liegt als in dem durch keine. gesetzlichen oder polizeilichen Bestimmungen verbotenen Zusatz von Wasser oder Brühwasser, der schon je nach dem benutzten Wurstrezept und der verwendeten Fleischqualität usw. sehr verschieden ist und der die Schmackhaftigkeit der Würste wenigstens bis zu einem gewissen Grade noch günstig beeinflussen kann. Über den Wert oder Unwert der Anwendung einer Verbältniszahl bei der Beurteilung des Wassergehaltes der Würste habe ich ${ }^{1}$ ) mich wiederholt so eingehend ausgesprochen, daß ich darüber keine weiteren Worte mehr verlieren möchte.

Soll also eine Wurst in jeder Hinsicht richtig beurteilt werden, so müssen ihre einzelnen Bestandteile erkannt und, soweit als möglich, quantitativ bestimmt werden. Den hierzu nötigen chemischen und mikroskopischen Untersuchungen sind häufig noch die Prüfungen auf Bindemittel (Stärke usw.), Frischhaltungsmittel, Farbstoffe und andere Verfälschungsmittel (Pferdefleisch, ekelhafte Fleisehteile usw.) anzuschließen, um Täuschungen bei der Beurteilung zu vermeiden. Auch auf Wurstersatzmittel, wie z. B. Ziegen- und Kaninchenfleisch, kann zu prüfen sein.

Man muf demnach eine ganze Reihe von chemischen und mikroskopischen Prüfungen ausführen, wenn man ein richtiges Bild von der Zusammensetzung und dem Wert einer Wurst gewinnen und ein unanfechtbares sachverständiges Gutacbten abgeben will. Dabei sind die wertvollen und wenigstens gesundheitsun schädlichen Bestandteile, also Art und Menge von Fett, Eiweiß, Wasser oder Brühwasser usw., gegenüber den wertlosen oder sogar ge fä hrlichen Bestandteilen unter Berücksichtigung

1) Chem.-Ztg. 1915, 39, 409 u. 431 und Diese Zeitschrift 1916, 32, 13 u. 29; 1918, 95, 393. 
des Preises der Wurst abzuwägen. Dieses Abwägen wird wesentlich erleichtert durch eine vernunftgemäße Heranziehung der bei der Nährwertberechnung sich ergebenden Werte, die wenigstens bezüglich des Heizwertes der Wurstwaren wertvolle Anhalts. punkte zu liefern vermögen.

Einige Beispiele mögen dies erläutern; da diese in der in Aussicht gestellten Abhandlung über die eingehende chemische Untersuchung der Wurstwaren ausführlich angegeben werden, kann auf ihre Wiedergabe an dieser Stelle verzichtet werden.

Jedenfalls ist zur Genüge bewiesen, daß zur gewissenhaften Beurteilung von Wurstwaren, namentlich in gerichtlichen Fällen, eine ausführliche chemische und mikroskopische Untersuchung dem sachverständigen Gutachten zugrunde gelegt. werden muls.

Der Vorsitzende: Wird zu den Referaten das Wort gewünscht?

\section{Diskussiun.}

Dr. Seel: Ich bitte, mir als dem Angegriffenen zu gestatten, die Diskussion zu eröffnen. Da habe ich zunächst zu sagen, dak die Feststellungen, die heute vorgeschlagen wurden, also bestimmte Zusätze von Wasser zu Ersatzwïrsten zuzulassen, von den Nahrungsmittelchemikern allein nicht gemacht werden können; denn die Überwachung der Wurstwarenherstellung bei den Kommunalverbänden und in den großen Fabriken rubt doch noch immer in den Händen der Tierärzte; ich glaube daher, daß es ohne die Mitwirkung der Tierärzte nicht möglich ist, in dieser Beziehung etwas zu erreichen (Oho! und Widersprach). Das ist me ine Ansicht. Ich will als Beispiel nur einen Fall erwähnen, in dem einer unserer Nahrungsmittelchemiker verschiedene Würste aus einem Militärschlachthaus auf Grund der Feder'schen Verhältniszahl beanstandet hat. Es ist $\mathrm{hm}$ dann von dem zuständjgen Kommandanten mitgeteilt worden, er möchte mit dem Material, das dem Schlachthaus zur Verfügung steht, selbst eine bessere Wurst machen; alle Offiziere und Mannschaften, welche die Würste bekommen hätten, wären damit sehr zufrieden gewesen. Die Würste waren sehr wässerig, dıs hing aber mit dem Material zusammen. I Ich habe den beaufsichtigenden Tierarzt gefragt, wie er sich zu der Frage stellen würde, wenn man bestimmte Mengen Wasser als erlaubt ansehen würde. Ja. sagte er, das kommt ganz auf das verwendete Warstbrät an; jedes Wurstfleisch ist anders beschaffen, und danach richtet sich der Wasserzusatz. Bis jetzt ist die Beaufsichtigung der Wurstherstellung leider nur in den Händen der Tierärzte, trotzdem ich schon vor Jahren den Vorschlag gemacht habe, daf3 sich die Nahrungsmittelchemiker am die Kenntnis der animalischen Nahrungsmittel etwas mehr als bisher bemühen möchten. Jedenfalis können wir ohne die Mitwirkung der Tierärzte in der Wurstfrage nicht gut za einer Entscheidung kommen, weil wir eben auf Schwierigkeiten stoken. Ich hatte erwartet, dak heute wenigstens die maßgebenden Tierärzte Berlins eingeladen sein würden; denn ohne diese können wir die Sache nicht machen. - Dann ist bei mir das zahlreicho Material bemängelt worden, das ich immer angekündigt, aber nicht veröffentlicht hätte. Ich habe ziemlich viel Untersuchungsergebnisse veröffentlicht. Ein anderer grofer Teil war auch bereits der Redaktion unserer Zeitschrift eingeschickt; es handelte sich da aber um Konserven, die in militärischen Anstalten gemaeht wurden; die Zeilschrift hat aber die Erlaubnis nicht erhalten, die Abhandlung in das neutrale Ausland za senden Auferdem habe ich noch eine ganze Menge Material von Wurst., besonders Kriegswurstuntersuchungen, das ich noch nicht veröffentlicht habe, weil gerade bei den schwierigen Untersuchungsverfahren noch Verbesserungen notwendig waren, die ich erst in der letzten Zeit habe machen lassen können, denn ich habe jetzt mehr zu tun als im Frieden. In der letzten Arbeit, die ich angekündigt habe, ist das alles genau ausgeführt, und ich möchte daher vorschlagen, daf man das Erscheinen dieser Arbeit abwartet. - Ferner möchte ich betr. der Verhältniszahl 4 diejenigen Herren bitten, welche in der Praxis Verhältniszablen bei Fleisch wie bei Wurst über 4 gefunden haben, dies doch mitzuteilen. Ich weik aus meiner Schlacht. haustätigkeit, daf jedes Fleisch rom Kopf bis zum Schwanz hinsichtlich. des Wassergehaltes ganz erheblich schwankt; die vordersten Teile sind die saftigsten und die besten. Nach dem jüdischen Gesetz werden ja nur die Vorderteile des Rindes als koscher angesehen, wahrend anf die trockenen Hinterteile mehr oder weniger verzichtet wird; daher kommt man, wie Herr Dr. Cantzler auch in seinem letzten Jahresberichte angegeben hat, bei einzelnen Fleiscbsorten zu Zahlen, die weit über 4 hinausgehen. Es ist mir auch von anderen Herren 
mitgeteilt worden, daß sie Verbältniszahlen von weit über 5 , sogar 5,8 in Fleisch gefunden bätten. Ich möchte die Herren bitten, dies heute hier zu bestätigen, wenn sie da sind. Jedenfalls 'bekommen wir in der Kriegszeit ganz andere Zahlen, wie in Friedenszeiten. Wer irgendwie Gelegenheit gehabt hat, Konservenfabriken zu besiehtigen und auch das dort zu schlachtende Vieh sich anzusehen, der wird zugeben müssen, daB die Qualitäten ron Jahr zu Jahr schlechter geworden sind; dabei können wir uns in Süddeutschland wohl einbilden, daki wix in dieser Beziehung vielleicht noch besser daran sind, als in Norddeutschland. - Was dıe übereinstimmenden Versuche anbetrifft, die mein Herr Vorredner angeführt hat, dak der eine Autor soviel gefunden hat und der andere soviel. so muf ich sagen: Die Übereinstim. mung dieser Zahlen findet ihre Erklärung dadurch, dab die Herren immer. Wurst untersucht haben, dje nach derselben Vorschrift hergestellt war und womöglich auch ungefähr von derselben Viehqualität; denn diese spielt dabei auch eine sehr grofe Rolle. Dak sich dann annähernd übereinstimmende Zahlen ergeben, ist selbstverständlich. Aber wir können beispielswelse nicht in ganz Deutschland, ja nicht einmal in einzelnen Landstricben annäbrend ubereinstimmende Zahlen bekommen. Ich muß ferner noch richtigstellen, wenn nämlich gesagt wurde, die Wurstherstellung geschähe jetzt fast durchweg fabrikmäfig. Ich babe hier eine grofe Arbeit vor mir, aus der hervorgeht, daf die Wurst in Norddeutschland meist fabrikmäßig hergestellt wird; für Süddentschland trifft dies aher nicht $z u_{\circ}$; dort gibt es gewiß auch Metzger, die fabrikmäßig arbeiten, wie in Norddeutschland, aber diese Fabrikwürste sind of schlechter als die Würste der kleinen Metzger. - So haben wir beispielsweise in Stuttgart die geschmacklosesten Würste, die äberhaupt nicht oder fast nicht gewürzt sind, aber die Bevölkerung will es so; unser Metzger z. B. weif genau, daß in Berlin besser gewürzt wird; dazu kommt, daf die kleinen Metzger bei uns alles ubriggebliebene Fleisch verwursten, in Norddeutschland ist das weniger oder gar nicht der Fall; in Süddentschland ist eben die Wurstbereitung hänfig noch ein Konservierungsverfahren für übrig gebliebenes Fleisch. Daher wird oft Fleisch verarbeitet, das viel trockener ist als das normale Fleisch. Ich habe selbst bei der Fleischbeschau oft die jüngen Bullen vor den anderen Tieren beschauen müssen, damil der Metzger das Bullentleisch gleich nach Hause bringen und fast noch blutwarm auf Wurst verarbeiten konnte, ein Kniff, der erlaubt ist und vom Staate ge* duldet wird; infolgedessen können wir nicht vorgehen, solange der Staat solche Kniffe gestattet and selbst wässerigere Würste macht, wie der Privaimetzger. Die Herren werden vielleicht anch Beispiele angeben können dafür, dass bei der Herstellung von Kommunalwürsten, von Standortwüsten und dergl. unter ihrer Aufsicht vielfach schlechter gearbeitet wird als von Privatmetzgern, und solange der Staat das duldet, kann der Privatmetzger deshalb nicht bestraft werden. Daß3 hier ein Wandel not tut, ist klar. - Der G elatinezusatz ist etwas, was in der Kriegszeit kaum mehr beanstandet werden kann, denn die Gelatine ist so teuer geworden, dafs sie kein Mensch mehr braucht. Der Metzger macht sich die notwendige Gelatine ans Bindegewebe selbst. Ich erinnere an einen Fall vor zwei Jahren, wo eine Leberwurst beanstandet wurde, weil sie Gelatine enthielt. Die Wurst wurde mit heikem Wasser ausgezogen; dabei hatte sich herausgestellt, daf der Auszug nichts anderes war als Leim bezw. Bindegewebe, das zu Leim verkocht war; diesen hatte der Nahrungsmittelchemiker ausgezogen, and weil er die leimgebenden Substanzen in der Wurst übersehen hatte, beanstandete er die Wurst wegen Gelatinezusatz; demnach war der Metzger unschuldig.' Jeden. falls sind in der Nahrungsmittelchemie wie bei der Fleischbeschau schon Fehler genug gemacht 'worden, sodas man vorsichtig sein mus bei der Herausgabe von irgendwelchen Forderungen oder Vorsehlägen betr. des Wasserzusatzes zu Würsten. - Eerner, ist gesagt worden: "Sämtliche Fachgenossen hätten sich auf den Standpunkt der Feder'schen Verhältniszahl gestellt. Dieser Vorwurf ist mir auch in einer Gerichtsverhandlung gemacht worden, obwohl dies gar nieht dahin gehörte: Ich kann diesen Vorwurf sofort dahin widerlegen, daf ich wohl der erste war, der dagegen geschrieben hat; ich war os aber auch nur deshalb, weil ich über die Sache gearbeitet habe und Gelegenheit gehabt babe, als Schlachthoftierarzt mir spezielle Kenntaisse daruber zu verschaffen. Ich habe die Sache aber in ganz kollegialer Weise zu erledigen versucht. Ich habe dem Kollegen erst ge* schrieben, später habe ich ihm mein Manuskript zugeschickt; wenn das fernerhin nicht mehr möglich gewesen war, so war das jedenfalls nicht meine Schuld. Ich wurde in der unglaublichsten Weise angegriffen, sodaß ich auf diese gehässigen Angrifte in den Zeitschriften nicht mehr antwortete. Kerner habe ich wiederholt festgestellt, daß sich bei den Würsten, für die ich so und soviele Prozent Wasser als Höchstgrenze angegeben hatte, dies alles auf die angefïbrten Lieferungsbedingungen beziehe, und daf bei diesen Lieferungsbedingungen das Vieh durchschnittlich gleichgute Qualität hatte, und dab das nur hierfur gelte, aber nicht beispielsweise für Wurst, die jetzt in der Kriegszeit hergestellt wurde. Das sind alles Sachen, die ich schon längst in der Zeitschrift widerlegt habe, trotzdom aber werden sie in den Ver. offentlichungen der Zeitschrift von anderer Seite wieder vorgebracht. Ich habe von vielen Fachgenossen aus Süddeutschland wiederholt die Nachricht erhalten, daß die Herren nicht nach der Feder'schen Verhältniszahl urteilen, sondern daß die einzelnen Kommunalverbände 
ihre Vorschriften selbst gemacht haben und daf man dabei sehr vorsichtig mit der Zahl 4 sein müsse, zumal meistens Höchstgrenzen für deu Wassergehalt vorgeschrieben seien, wie früher in den Vereinbarungen. - Daß ich Tabellen äber den Wassergehalt einzelner Fleischteile vollständig in einer Voröffentlichung angeführt habe, kann nicht ein Grund zu einer Beanstandung oder gar Lächerlichmachung sein. Wenn ich derartige Listen wie diejenige von Abderhalden angeführt habe, so ist dies mit Absicht geschehen, um einen vollständigen Überblick über den Wassergehalt der einzelnen Organe zu geben. Das Beispiel von den "Augen" ist schon deshalb nicht zutreffend, weil die Augen, wie ich schon früber angegeben habe, za den verbotenen Wurstbestandteilen gehören. Im Schlachthaus wird jedes Auge bei der Beschan. sofort vernichtet, denn wenn ein Metzger ein Auge bekommen kann, so besteht Gefahr, daß es eben mitverwurstet wird.

Der Vorsitzende: Ich habe nicht den Lindruck, dak Sie läeherlich gemacht werden sollten. Ich bitte, nur auf das einzugehen, was Kollege $\mathrm{F}$ ed er gesagt hat.

Dr. Seel: Ich habe bei Lebor würsten nachgewiesen, dás gerade die Leberwürste, die bei uns nach Berliner Art gemacht werden, eine Verhältniszahl von unter 3 haben. Wenn man solche Leberwürste beurteilen will, kann man nicht die Verhältniszahl 4 zugrunde legen, hier ist sie an und für sich viel zu hoch. Die gewöhnlichen Leberwürste dagegen, die nach süddeutschem Geschmacke magere Organe and alles mögliche, besonders Bindege webssubstanzen enthalten, sind natürlich viel minderwertiger und haben auch eine höhere Verhältniszahl und einen höheren Wassergehalt als die fetten Berliner Streichleberwürste. Das wäre so ziemlich das hauptsächlichste, was jch zu den Ausführungen des Herrn Feder zu sagen hahe. Für jede Vorschrift und für jede Sorte ist also eigentlich eine besondere Verbaltniszahl nötig; infolgedessen kann man mit der $\mathrm{F}$ eder'schen Verhältniszahl überhaupt nicht arbeiten.

Der Vorsitzende: Zur Förderung der Diskussion möchte ich folgendes bemerken: Es handelt sich - und ich bitte die Herren, bei ihren Ausführungen darauf Rücksicht zu nehmen - um zwei Fragen: Ist es zulässig, einen bestimmten Wasserzusatz festzasetzen und ist die. Angabe der Fed er'schen Vorhältniszahl richtig? Daran bitte ich die Herren sich in der Diskussion zu halten.

Geheimrat Juckenack: Grundsätzlich werden natürlich alle die Fälle ausscheiden müssen, in denen auf Anordnung irgendeiner kommunalen oder staatlichen Behörde irgendwelche besonderen Wurstwaren angefertigt werden. Es kann sich hier nur darum handeln, wie wir uns in den Fällen zu verhalten haben, wo lediglieh das Nahrungsmittelgesetz in Frage kommt.

Der Vorsitzende: Ich glaube, das sind die beiden Hauptpunkte, auf die es zunächst allein ankommt. Ich bitte, sich nicht allzusehr in Einzelheiten za verlieren und anch nicht persönliche Angriffe auf den einen oder anderen zum Gegenstand der Erörterung zu machen, denn dabei kommt nichts heraus, sondern sich auf die Kernpunkte zu beschränken, und die sind: Ist ein Wasserzusatz zulässig und stimmen die Angaben der F e de r'schen Verhältniszahl?

Prof. Behre: Es wäre ganz gewiß sehr erfreulich und zu begrüßeń, wenn es möglich wäre, den Wassergehalt der Würste nicht nur richtig zu bestimmen, sondern ihn auch so festzulegen, daß wir in der Praxis unser Urteil daraaf gründen können. Nun fürchte ich aber, daß das nicht möglich ist. Wenn die Reichsfleischstelle den Fleischerinnungen Anweisungen gibt, in welcher Weise sie ihre Wurst herstellen können, und wenn die Reichsstelle ihnen, wie ich hier ans diesen Anweisungen an verschiedene Innungen feststellen muf, $16 \% \mathrm{~W}$ asserzasatz bei Blatwurst und $36 \%$ Wasserzusatz bei Leberwurst anheimgibt, dann kann von unserer Seite nicht gut etwas anderes, beschlossen werden. Das halte ich für den wichtigsten Punkt, weshalb wir über den Wasserzusatz zu sog. rationierten Wurstwaren Bestimmungen vorläufig nicht treffen können. - Dann ein anderer Punkt, nämlich der Wassergehalt des Fleisches selbst. Ich "bin wiederbolt in den Veröffentlichungen über diesen Gegenstand mit als Kronzeuge benannt worden, daif der Wassergehalt des Fleisches nicht den Grenzen entspricht, die Kollege $\mathrm{Feder}$ in seinen ersten Veröffentlichungen angegeben hat. Wenn ich mich mit dieser Frage béschäftigt habe, so hat das den Grund, daf ich bei der Zusammenstellung der bisherigen Untersuchungsergebnisse gefunden habe, daf doeh eigentlich recht wenig Untersuchungsmaterial über diesen Gegenstand vorhanden ist. Wenn man alle die Proben einmal zusammenzählt, die bisher untersucht worden sind, und worauf die Grenzzahl von Feder sich aufbaut, so sind auber den 247 Proben, die seinerzeit in dem Buch ${ }_{\text {Die }}$ mensehlichen Nahrungs- und Genufimittel" von J. König zusammengetragen worden sind, soviel ich bisher ersehen kounte, überhaupt nur 64 Proben Filcisch tntersucht worden, und aus diesen $64+247$ Proben Fleisch ist dann eine Verhältmiszahl von bis zu 3,8 herausgezogen worden. In meinem Amte sind in der Zeit von 1917 bis 1918 insgesamt 18 Proben Fleisch untersucht worden, dabei haben acht Proben eine Verhältniszahl von über 4 und zwei von über 4,5 ergeben. Das mag vielleicht besondere Gründe haben. Es ist nicht ausgeschlossen, daß $B$ in Chemnitz jetzt vielfach geringwertiges Vieh geschlachtet wird, wobei Fleisch gewonnen wird, das anormale Zahlen ergibt; jch halte das nicht für ansgeschlossen. Aber wenn das der Fall ist - und 
die Zahlen stimmen ja - dann mübten meiner Ansicht nach noch mehr Untersuchungen gemacht werden, um mit Sicherheit festzustellen, in welchem Umfange die Fe der'sehen Sehlakfolgerungen richtig sind. Ioh würde also in dieser Beziehung empfehlen, bei der Anwendung der Feder'schen Verhältniszahl 4 zum mindesten vorsìchtig zu sein, vor allen Dingen in der Kriegszeit. Ich würde weiter empfehl $n$ n, noch mehr Untersuchungen in verschiedenen Teilen des Reiches zu machen, nm festzustellen, ob die Verhältniszabl von 4 uberall als das Höchstmaßs angesehen werden kann. Für Chemnitz, wie gesagt, ist dis nicht der Fall. Ich will zum Schlúf noch die Zahlen anführen, die gefunden wurden bei Proben, die auf dem Schlachthof entnommen worden sind: 4,98, 4,29, 4,08, 4,04, 4,25, 4,47 und 4,80. Das sind immerbin Zahlen, die Anlaf3 zu Bedenken geben und die die Anregung geben sollten, noch mebr Untersuchungen in dieser Richtung auszuführen. Dabei wäre Fleisch von verschiedenen Körperteilen und auch sogenanntes Ausputzfleisch zu untersuchen. Auch Gefrierfleisch ist von uns in dieser Richtung geprült worden. Über das Ergebnis aller dieser Untersuchungen werde ich demnächst in unserer Zeitschrift berichten.

Dr. Leonhard: Ich wollte zunächst dazu das Wort ergreifen, daß, wenn man der Sache auf den Grund geht, Gegensätze zwischen den Herren Seel und Feder gar nicht vorhanden sind. Es ist ganz selbstverständlich, daß, wenn pine Wurst die Fe de r'sche Verhältniszahl aufwoist, damit noch lange nieht gesagt ist, daß sie auch in allen anderen Punkten den Bestimmungen des Nahrungsmittelgesetzes und der ortsüblichen Herstellungsweise entspricht. Die Feder'sche Zahl will nichts sein als eine Beurteilung der Wurstwaren in bezug auf den Wassergehalt und für diesen Punkt ist sie nach meinen selrr reichlichen Erfahrungen auf diesem Gebiet in ihrer Grundiage sicher zutreffend. Die Ansicht des Kollegen Seel, dak zur Beurteilung auch die Art des verwendeten Rohmaterials heranzuziehen ist, ist ebenfalls etwas Selbstverständliches und steht nicht im germusten Gegensatz zu den Fe de r'sehen Ausführungen. Ich stehe nicht an zu erk]ären, dás anch mir eine Wurst, die $20 \%$ Wasserzusatz enthält, lieber ist. und wertvoller erscheint, als. eine Wurst, die kein zugesetztes Wasser enthält, aber ausschlieblich aus Knorpel und Bindegewebe und anderen Abfillstoffen besteht. Eine derart hergestellte Wurst kann jedoch jederzeit, auch wenn sie die Feder'sche Verhältniszahl hält, anf Grund des Nahrungsmittelgesetzes beanstandet werden. Ich babe bereits im Jahre 1913 sebr eingehende Ontersuchungen über Wärste gemacht, deren Beurteilung im Grunde anf die Feder'sche Verhältniszahl hinausläuft, wenn diese Beurteilung auch damals nicht in der Verhältniszahl ausgedrückt wurde. Ich habe nur eine eirzige Einwendung gegen die Zulassung der Feder'schen Verbältniszahl zu machen, nämlich bei der Beurteilung von Blutwärsten; da ist mir die Fed er'sche Verhältniszahl allerdings nicht verständlich, denn bei den Blutuntersuchungen, die wir vorgenommen haben, haben wir festgestellt, dak der Wassergehalt des Blutes etwa $82 \%$ beträgt. Außerdem gibt das Blut ganz erhebliche Mengen Bestandteile bei der Ätherextraktion ab, und dies muß bei der Berechnung der fettfreien Trockenmasué in Betracht gezogen werden, sodaf ein fettfreier Trockenrückstand des Blates nach unseren Untersuchungen mit 16,5-17\% anzunehmen ist. Es ist mir nicht recht verständlich, wie Feder anch bei Blut zur Verhältniszahl von 4 kommt; er wïrde richtiger die Verhältniszahl anf etwa 5,5 berechnen. - Dann möchte ich noch eines bemerken: Kollege $\mathrm{F}$ ed er hat hingewiesen auf die Angaben, die ich in meinem letzten Jahresbericht gemacht habe, dak ich aus dem Grunde die Beurteilung in der. Weise vornehme, daE jch angebe: Zur Herstwllung von $1000 \mathrm{~g}$ Wurst sind verwendet worden: a $g$ ursprüngliches fettfreies Fleisch, b $g$ Fett, c $\mathrm{g}$ Kochsalz unter Zusatz von $x$ Teilen Wasser, weil diese Aufstellung für den Nichtfachmann viel leichter verständlich ist, als die Feder'sche Verhältniszahl. Es macht außerordentliche Schwierigkeiten, wie ich in verschiedenen Verbandlungen gesehen habe, dem Richter und namentlich dem Laienrichter das Wesen der Feder'schen Verhältniszahl klar zu machen, während er sich ohne weitc res klar ist, wenn ihm gesagt wird: Zur Herstellung der Wurst sind die und die Mengen Rohmaterial oder folgende Zusätze verwendet worden. Ob $f$ s möglich sein wird, heate schon eine Grenze für den Wasserzusatz der Würste festzusetzen, - ich bezweifle es; es herrschen in dieser Beziehung anßerordentlich verschiedene Verhältnisse in den verschiedenen Gegenden. Die Würste werden selbst in verschiedenen Orten desselben Bezirks auf recht verschiedene Art hergestellt. W's muß da unbedingt pach meiner Auffassung zuerst ein erbehliches Zahlenmaterial vorliegen, damit Festsetzungen getroffen werden können. Auf Grund der Untersuchungen, die wir gemacht haben, kann ich aher bestätigen, was Kollege Feder gesagt hat, dak, wenn nach der Verhaltniszahl sich ein Wassergehalt von $10 \%$ berechnet, dak man dann mit rubigem Gewissen sagen kann, da 6 mindestens $15 \%$ Wasser zugesetzt worden sind, sodaf: also die Verbültniszahl in den Fällen, die mir bisher zur Beurteilung vorgelegt worden sind und wo ich das habe genau übersehen können, stets ein für den Metzger außerordentlich günstiges Ergebnis zeigte, sodaß nach meiner Ansicbt eine Täuschung zu ungunsten des Metzgers bei Anwendang der Feder'schen Zahlen ausgeschlossen ersiheint. Ich mörhto aber doch bitten, dafs erst festgestellt wird, in weleher Menge oder in welcher üblichen Weise das Wasser beim Matzger zugesetzt wird. Wir haben in Heidelberg Metzger, die bis 400 und 500 
Teile Wasser auf die gleiche Menge Wurst zusetzen. Ich halte dies nicht für erlaubt, aber ich wollte nur aufuhren, dẩ das Brauch ist, oder das der Brauch oder Mifbrauch bei nus eingerissen ist; es muß aber jedenfalls doch bei der Festsetzung der Grenze einigermaken den ortsüblichen Gewohnheiten Rechnung getragen werden; ich weif auch nicht, ob wir hente sehon in der Laye sind, bestimmte Grenzen festzusetzen.

Prof. Buttenberg: Es wurde vorgetragen, daf die Fettbestimmung in Fleisch. nach Soxhlet ausgeführt worden ist. Man kommt viel schneller vorwärts, wenn man die Wurstmasse mit Salzsäure anfschliekt und im Gottlieb'schen Rohr ausschüttelt. Diese Arbeitsweise hat sich in unserm Betriebe bei Massenuntersuchungen sehr bewahrt.

Dr. Fend ler: Die Festsetzung eines höchitzulässigen Wasser der Ersatzwärste hat vieles für sich. An sich erscheint die Forderung durchaus berechtigt. daf der Wasserzusatz die technisch notwendige Menge nicht überschreiten solle. Der Zweck dieser Fordernng ist der Schutz des Verbrauchers vor Übervorteilung; ubervorteilt wird er, wenn er gezwungen ist, stark wasserhaltige Wurst zu einem Preise zu kaufen, der nur für wasserärmere Wurst angemessen wäre. Stark wasserhaltige Wurst ist in bezug auf ihren Nährwert verschlechtert. Bei den Preisen, die für Ersátzwürste heute leider angelegt werden müssen, besitzen diese jeloch weniger den Charakter eines Nahrungsmittels als denjenigen fines Genufsmittels. Bie haben in erster Linie die Bestimmung, eine Lijcke in dem Mangel an Brotbelag- und Aufstrichmitteln auszufüllen, das Brot schmackhafter zu machen und somit eine kleine Abwechselung in die einförmige Beköstigung zu tragen. Nur wenigen Begüterten wird es gèstattet sein; Ersatzwurst in solchen Mengen zu genieben, daf man von der Zuführung erheblicher Nährwerte sprechen kann. Betrachtet man die Angelegenheit unter diesem Gesichtspunkte, so wird sich die Erage ergeben, ob es nicht zweckmäfisig ist, durch die Zulassung eines höheren Wassergehaltes, als des technisch notwendigen, eine Verbilligung dieses Genufmittels za bewirken. Die Kaninchen- und Zieqenwürste spielen auf dem Berliner Markte eine grobe Rolle. Infolgedessen mufte die Ersatzmittelstelle Grok-Berlin sich eingehend mit ihnen befussen. Sie hat lange die Frage erörtert, ob die Zulassung derartjger Ersatzwürste in ihrem Zuständigkeitsgebiete nicht grundsätzlich versagt werden sollte. Neben anderen Gründen. wilche hier im Augenblick nicht interessieren, war hierbei der Gesichtspunkt makgebend, dak durch die Fubrikation von Ersatiwürsten dem Markte markenfreies Fleisch entzogen werde. Dieser Gesichtspunkt wurde jedoch fallen gelassen, da bei einem Ausschluf der Ersatzwurstfabrikation aus dem Gebiete Grok Berlins die Kaninchen und Ziegen voraussichtlich nach andern Gehieten abgewandert wären, welche sich auf einen weniger ablehnenden Standpunkt stellen. Wenn schlieblich der Beschluf gefaft wurde, derartige Würste zu genehmigen, so geschah dies unter dem Gesichtspunkte, daß ein dringendes Bedürfnis für derartige Brotbelagund Aufstrichmittel besteht. Als Voraussetzung wurde jedoch geltend gemacht, daf die Ersatzwürste dem Markte zu Preisen zugeführt werden, welche den gelegentlichen Erwerb dieses Genufmittels auch den weniger Begüterten ermöglichen. Bei don hohen Preisen, welche hente für Kaninchen und Ziegen angelegt werden müssen, erscheint eine verhältnismäfige Niedrighaltung der Preise nur durch zwei Maśnahmen möglich.' Erstens soll die Herstellung nur dann zugelassen werden, wenn der Hersteller keine übertrieben bohen Preise für das Rohmaterial anlegt, zweitens soll durch die Zulassung eines höheren als des technisch notwenigen Wasserzusatzes eine Streckung erfolgen Unter diesem Gesichtspunkte hat die Ersatzmittel. stelle Grof-Berlin sich auf Grund eingehend erörterter Kalkalationen Richtpreise gebildet, bei deren Überschreitung eine Genehmigung nicht mehr erfolgt, auch hat sie zugelassen, daf bis zu 40 Telle Wasser oder Brühe anf 60 Teile Fleisch und Kramteile verwendet werden. Die Preise sind selbstverständlich dem Wasserzusatz entsprechend kalkuliert worden. Durch eine Bekanntmachung ist der Verbraucherkreis über den zugelassenen Wasserzusatz unterrichtet worden. Derartige Würste, welche unter der Aufsicht eines Kollegen hergestellt wurden, waren von ausreichender Qualitä. Der Wassergehalt beträgt nach den angestellten Versuchen bei Jagdwurst nicht mehr als 80 , bei Leberwurst nicht mebr als $75 \%$. Diese Wassergehalte sind hoch. Sie entsprechen nicht den Anforderungen, welche wir an Friedenswürste zu stellen gewohnt sind, doch habe ich auch von meinem Standpunkt als Nahrungsmittelchemiker mich nach reiflicher uberlegung genötigt gesehen, dieser Kriegsmafnahme zuznstimmen. Daf es sich nur um eine Kriegsmaßnahme handelt, glaube ich besonders betonen zu müssen; ich halte für selbstverständlich, daß3 bei einer Besserung der allgemeinen Verhältnisse des Nahrungsmittelmarktes auch hier ein Abbau stattfinden wird.

Dr. Willeke: Wir haben uns auch mit der Prüfung und Zulassung von markenfreier Fleischwurst eingehend befassen müssen. Wir sind aus den gleichen Erwägungen, die eben von dem Herrn Vortragenden vorgebracht worden sind, zu der Zalassung gekommen, und zwar auch nur mit einer Begrenzung des höchstzulässigen Wassergehaltes und des Preises. Allerdings, zu den hohen Preisen siud wir nicht gekommen nud auch nicht zu den hohen Wasserzusätzen wie sie an anderen Orten genehmigt sind. Die Wasserzusätze haben wir in gleicher Höhe bemessen, wie sie für die kommunale Wurstherstellung vorgeschrieben waren: 
Auf hundert Teile Fleischmasse 30 Teile Wasser, das wären ungefähr 25\%. Die ganze Frage der zulässigen $\mathrm{W}$ assermenge ist im Grunde nur eine Preisfrage. Also, wenn es nicht möglich ist, îberhaupt abzusehen von der Herstellung markenfreier Wurst - und auf seiten der süddeutschen Stellen scheint ja die Absicht zu bestehen, gegen die Zulassung von markenfreier Wurst anzukämpfen - müssen wir unbedingt den Wasserzusatz beschränken. Eine beliebige Höhe des Wasserzusatzes bei solcher Wurst kann unmöglich zugelaszen werden. Ich will Ihnen das an einem Beispiel zeigen. Die Frankfurter Konservenwürste waren früher nach der Lokalverordnung nicht genehmigungsptlichtig. Bei diesen Würsten hat sich ergeben, daßs sie kurz nach dèr Herstellung pro Paar $100 \mathrm{~g}$ wogen, bei der Prüfung im Laboratoriam aber nur noch $70 \mathrm{~g}$, während Würste gleicher Herstellung, die zu gleicher Zeit auf einem Bahnhof beanstandet wurden, gar nur $50 \mathrm{~g}$ schwer waren. Das Paar Würstchen war also von 100 auf $50 \mathrm{~g}$ eingetrocknet. Der Wasserzusatz betrug hier nach unserem vom Herrn Vortragenden auf meine Veranlassung mitbeigebrachten Material $50 \%$ und mehr. Das bedeutet natürlich eine Übervorteilung der Käufer, die auf keinen Fall als zalässig angesehen werden kann. Das ist ein Schwindel, und die Preise für Kaninchen und Ziegen usw. sind nur durch derartige gewinnbringende Verarbeitung zu Wurst mit so hohen Wasserzusätzen so hoch getrieben worden. Subald die Herstellung ganz unterbunden würde, würde der Preis zweifellos genau wieder so sinken, wie er für Roßffleisch und andere Fleiseharten gesunken ist. Also, grundsätzlich wird davon auszugehen sein: Wenn man die Herstellung von Wurst sus markenfreiem Fleisch tiberhaupt genehmigen will, muß man unbedingt einen höchstzulässigen Wasserzusatz vorschreiben. leh bin der Meinung, nach unseren Erfahrungen $k \cdot a n n$ man auch einen solchen höchstzulässigen Wasserzusatz vorschreiben, weil man mit Hilfe der Verhältniszahl von Feder doch die genügende Sicherheit hat, festzustellen, ob er nicht erheblich überschritten worden ist.

Prof. Rupp: Die vorzüglichen Arbeiten äber den Wassergehalt in Wurstwaren und die Verbaltniszahlen sind außerordentlich dankenswert, aber nach meiner Ansicht ist die Frage des Wassergehaltes in Wurstwaren noeh nicht spruchreif, und zwar aus folgenden Grtinden: Wie schon erwähnt, ist die Art der Wurstherstellung durchaus verschieden, sowohl nach den einzelnen Ländern, wie nach den einzelnen Bezirken. Ich erinnere daran: Früher war es in Säddeutschland nicht äblich, Stärkemehl zar Wursttabrikation zu verwenden, während es in Norddentschland in manchen Gegenden jetzt noch ortsüblich ist, Stärke bei der Wurstfabrikation zu verwenden. Wir müssen namentlich bedenken, daßs wir jetzt in der Kriegszeit woder das normale Rohmaterial, Fleisch und die sog. Schlachtabgänge, worauf es auch ganz besonders ankommt, zur Verfügung haben, noeh nornale Arbeitskräfte besitzen. Wor macht denn heute die Würste. wenn man von den Wurstfabriken absieht? Geben Sie in die länd. lichen Bezirke, dort fehlen die nötigen Arbeitskräfte; sie stehen im Felde und es müssen Lehrlinge und solche, die vielleicht niemals in threm Leben Wurst fabriziert haben, zu dieser Arbeit herangezogen werden. Es schwebt mir, auch gerade beztiglich der verschiedenen Arten der Wurstbereitung, ein recht guter Witz der "Fliegenden Blätter" aus der letzten Zeit vor; wo der Lehrling beim Würstemachen zu seinem Meister sagt: „Meister, wenn das aber rauskommt, was da alles reinkommt!" (Heiterkeit.) Ich will damit sagen: Die Wurstbereitung ist in den einzelnen Bezirken so versehieden, daßs wir jetzt eine Grenzzahl nicht feststellen können, sondern daß wir vielleicht gut tun, unsere Kollegen anzuregen, in der Friedenszeit Arbeiten über den Wassergehalt der Wurstwaren und den Zusatz von Wasser bei der Wurstbereitung anzustellen, um dann vielleicht erst der Frage näher zu treten, ob es möglich ist, eine Grenze für den Wassergehalt der Wurstwaren festzusetzen.

Dr. Rothenfuber: Ich möchte anschließend an die Ausführungen des Herrn Vorredners mitteilen, daß̈ es in Bayern diese Zubereilungen wie Kaninchenwürste, Jagdwürste, Ziegenwürste und was da alles aufgekommen ist, eine lange Zeit überhaupt nicht gegeben hat. Auf einmal kamen sie auf, und zwar auch gleich zu den hohen Preisen von 6, 7 und $8 \mathrm{M}$. wie bei Ihnen; das hat allerdings nicht lange gedauert; die Preise gingen zunächst wieder auf $6 \mathrm{M}$. zuräck, und dann warden Höchstpreise festgesetzt - ich glaube, ich weiß es nicht genau, von $4,50 \mathrm{M}$. - und da sind diese Würste natürlich sehr schnell wieder verschwunden, wie zu erwarten war; schlieflich wurde auch eine Art Bewilligung beschränkter Herstellung durchgeführt - die Würste durften überhanpt nur mehr in bestimmten Schläehtereien hergestellt werden - und die Folge war, dak nur noch sehr wenig davon zu sehen war und zu verhältnismäßig billigen Preisen. Nun kommt die Gruidfrage: Warum werden diese Würste überhaupt hergestellt? Die Lösung liegt in dem, was ich eben gesagt habe: Sie werden nur hergestelli, um viel Geld zu machen. Wenn es möglich wäre, diese Würste inberhaupt aus der Welt zu schaffen und das Ziegen- und Kaninchenfleisch unmittelbar zu verkaufen, so wäre das viel besser; ich habe mal selbst einen solchen Schlächter gefragt: Warum machen Sie überhaupt solche Würste? Er antwortete: An Ziegenfleiseh und Kaninchenfleisch verdient man nicht genug"; die Folge ist also, daß́ Kaninchen- und Zjegenfleisch überhaupt nur in Form dieser teuren Würste zur Verfügung steht. Was den Wassergehalt betrifft, 
so glaube ich aus den bisher geführten Ausführungen entnehmen zu können, daßs doch noch nicht genug kirfahrungen vorliegen, um eine derartige Verhältniszahl festzusetzen. Im übrigen möchte ich noch einmal betonen: Es wäre wünschenswert, diese Würste uberhaupt aus der Welt zu schaffen und dafür das Fleisch als solches zu verkaufen; wir wärden damit jedenfalls besser fahren.

Dr. Kortenbach: Ich möchte erwähnen, daß schon im Winter 1914/15 in Duisburg sehr wässerige Wurst in den Verkehr kam; sofort habe ich mich mit der Sache befaft und festgestellt, dak ein ganz enormer Wasserzusatz erfolgt war and zwar mit einer Verhältniszahl von 5-6. Nachdem ddraufhin einige Bestrafungen vorgekommen waren, ging der Wassergehalt sofort zurück. Ich habe eine groke Menge Material in Händen gehabt und habe dabei sofort sehen können, daß die durch die F' e d e r'sche Verbältniszahl verlangten Forderungen tatsächlich durchaus berechtigt waren. Vom Mai 1915 ab wurde bei uns die ganze Wursterei in Kommunalverwaltung genommen; ich habe dann eine ständige Überwachung vorgenommen und habe ein Material gehabt von 600-700 Würsten; im allgemeinen habe ich eine Verbältniszahl von nicht über 4,6 erreicht. Unter Berücksichtigung der aligemeinen Verhältnisse und auch, um möglichst viel Wurst zu bekommen, haben wir Zahlen von 4,6 durchgelassen. Sobald höhere Zahlen vorkamen, habe ich es moniert, und warde die Angelegenheit dann auch geregelt. Auch in anderen Städten; für die wir die Untersuchung ebenfalls ausgeführt haben, habe ich die Forderung gestellt und erreicht. In einem Falle hat mir der Wurstobermeister, wie ich ihm sagte: "Hören Sie mal, die Wurst ist aber doch zu arg gewässert" erklärt: „Ja, Sie haben recht; wir haben diesmal bedeutend mehr Wasser zugesetzt wio sonst". Gute Würste habe ich von den Landkreisen bekommen, wo noch kleinere Metzger selbständig die Wursterei inne hatten, die von der Kommune das Fleisch bekommen hatten, und habe daraus die Überzeagung gewonnen, daf die Beurteilung des übermäfigen Wasserzusatzes in Würsten auf Grund des Feder'sehen Verfahrens durchaus zu Recht besteht.

Dr. Mezger: Wir haben in Stuttgart eine groGe Reihe von Kriegswürsten hinsichtlich der Fe de r'schen Verbältniszahl geprüft; wir haben in sehr vielen Fallen eine über 4 liegende Verbältniszahl gefunden, aber wir haben uns bis jetzt nicht dazu entschliefen können, einen dieser Falle vor Gericht zu bringen. Ich habe mich darauf beschränkt, die Metzger auf den übermäßigen Wasserzusatz hinzoweisen, und zwar deshalb, weil wir feststellen konnten, daf auch die unter amtlicher Aufsicht hergestellten Würste die Verhältniszahl offters nicht einhielten; infolgedessen babe ich mich nicht entschließen können, das Odium auf mich zu nehmen, daf die eine Behörde an dem Strange zieht und die andere an dem anderen. In der Sache selbst bin ich der Ansicht, daß die Frage für uns jetzt in der Kriegszeit noch nicht spruchreif ist. Bei uns anf dem Lande sind es jetzt vielfach die Frauen, die die Wurstherstellung betreiben müssen, und ob die das Gewerbe so gut verstehen wie ihre Männer, weifs ich nicht; jedenfalls möehte ich dringend zur Vorsicht mahnen, ehe wir eine Entschliefang fassen.

Prof. Beythien: Mir scheint auch, wie einer der Herren Vorredner schon ausgefuhrt hat, daß zwischen den Kollegen Feder und Seel ein grundsätzlicher Zwist gar nicht besteht, dafs sie eigentlich gute Freunde sind. Es scheint mir allerdings ein Mißverständnis auf seiten des Herm Kollegen Seel vorzaliegen, denn ich verstehe die Ausführungen des Herrn Feder nicht so, daf er der Meinung ist, wenn nur der Wassergehalt in Ordnung ist, so ist die ganze Wurst normal, gleichgültig was sonst darin ist. So war es wohl nicht gemeint, er wollte nur einen besonderen Teil der Wurstuntersuchung herausgreifen, und hat das meiner Ansicht nach allerdings in mustergültiger und erschöpfender Weise getan. Die Schwierigkeiten, die seiner Aufgabe entgegenstehen, sind uns bekannt; die treten uns bei den Untersuchungen sämtlicher Nahrungsmittel immer entgegen. Das Verfahren, das Herr Seel im Auge hat zur genauen Bestimmung des Wassergehalts, ist theorotis ch zweifellos richtig. Wir müssen mit Hilfe des Mikroskops zunächst feststellen: Welche tierischen Gewebe sind in der Wurst vorhanden. Kollege Seel hat aber übersehen, dás diese Feststellung noch nicht genügt; denn wir müssen auferdem wissen, wieviel von jeder Art tierischen Gewebes in der Wurst ist. Wir müssen also mit Hilfe des Mikroskops eine quantitative Ermittelung vornehmen, wieviel Sehnen, Augen, Nägel usw. (Hei erkeit) darin sind - alles das muß festgestellt werden und für jeden einzelnen Bestandteil ist dann der zugehörige Wassergehalt in Ansatz zu bringen. Wenn wir mit der Bekämpfung des übermäbigen Wasserzusatzes warten wollten, bis diese Aufgabe gelöst ist - das ist die Quadratur des Zirkels gewissermaken - so wird keiner von uns das Ende erleben. Kollege Seel schätzt uns ja nicht sehr hoch ein auf diesem Gebiet, aber wir sind doch alle mehr oder weniger eingeweiht in die Mikroskopie, und die mikroskopisehen Kenntnisse der Tierärzte sind doch auch noch nicht allzuweit gediehen (Heiterkeit); auch die Tierärzte können nicht jedes einzelne Gewebe mit Sicherheit von den anderen unterscheiden. Es wird Herrn Se el auch gegangen sein wie mir, daß er darüber eimige Lirfahrungen sammeln konnte. Mich hat es geradeza schmerzlich berührt, daf Herr Seel uns die Grenzen unseres Könnens, wie mir scheint, etwas lieblos unter die Nase gerieben hat. Wir können im allgemeinen wohl sagen, wir sind durchàrungen von der Unzulanglichbeit unseres Wissens, 
und es tritt bei uns in jeder Versammlang bei jedem einzelnen Sprecher eine sehr scharfe Selbstkritik hervor; demgegenüber kann man wohl feststellen, daf den Tierärzten eine solche Selbstkritik nicht zur Last zu legen ist. Im Gegenteil, sie kranken in der Regel an einer starken Überschätzung ihres Könnens; sie mischen sich, wie uns allen bekannt ist, in unsere Kreise hinein, sie suchen sich in Arbeitsgebiete, die wir als unsere ureigensten Gebiete zu betrachten und zu schätzen gewohnt sind, einzudrängen und uns daraus zu verdrängen. - Nicht immer aus rein idealen Gründen, und deshalb hat es mich sehr schmerzlich berührt, daf dieser Gegensatz gerilde heute hier zur Sprache gekommen ist. - Was nun die Frage des Wassergehalıs selbst betrifft, so würde ich es auferordentlich begrüBen, wenn die Arbeiten und die Anregungen des Herm $\mathrm{Feder}$ heute nicht ganz im Sande verlefen. Ich verkenne ja nicht, daß gerade die jetzige Kriegszeit nicht besonders geeignet ist, um die $F^{\prime}$ eder'sche Zahl anf ibre absolute Richtıgkeit zu prüfen. Die Kriegsverhältnisse bedingén tatsächlich, daß das Material zur Wurstherstellung doch wahrscheinlich ein wesentlich anderes ist, wie es in normalen Zeiten gewesen ist. Ich würde aber gar kein Bedenken haben, dak wir uns dahin äufern, daf diese Grundsätze Feder's unsere Billigung finden, daß wir die Grandlagen seines Verfahrens an und für sich für richtig halten und uns nur vorbehalten, noch weitere Untersuchungen tuber die Grenzen der Verhältniszahl anzustellen, wie wir das bei allen unseren bisherigen Vereinbarungen gemacht haben. - Ich erinnere an unsere Fruchtsaft-Statistik und ähnliche Fragen. Diese aufschiebende Haltung bei den Fruchtsäften hat uns nicht gehindert, grobe Wasserzusätze beim Himbeersirup $\mathrm{zu}$ beseitigen, und ich glaube, wir sind sehr wohl in der Lage, grobe Verfälschungen der Wurst mit Wasser schon jetzt $\mathrm{zu}$ bekämpfen. $\mathrm{Ob}$ es nun zweckmäßig ist, die Wurst, die gegen Marken, also in normalen Handel abgegeben wird, hinsichtlich des Wassergehalts zu begrenzen, diese Frage läft sich sehwer beantworten. Hier spielt wieder das Thema der Autosuggestion hinein, das wir schon mehrfaeh gestreift haben - hierher gehört ja auch die Hersteliung des zu 94\% ausgemahlenen Brotes, oder vielmehr des Brotes aus so hoch ansgemahlenem Getreide - und aus dem gleichen Grunde mag es vielleicht $z$ weckmäßin erscheinen, den Leuten zunächst mal ein möglichst großes Stück Wurst zu geben. Bei der Warst, die im reellen Handel abgegeben wird, ist die Gefahr des Betrugs ja auch nicht oo grof; dringend erwünscht scheint mir aber, daf die sogenannte markenfreie Wurst sehr scharf beobachtet wird, und hier trıfft auch nicht $2 u$, was als Bedenken geäufert worden ist, dafs die Wurstbereitung in den verschiedenen Gegenden Deutschlands verschieden ist. Mit der Kaninchen. and Ziegenwurst braucht sich der einfache Fleischer gar nicht zu beschäftigen, wenn er sie nicht in einer verhaltnismaffig anständigen Weise herstellen kann, und da scheinen mir allerdings die Versuche von Feder sehr ausschlaggebend zu sein. Er hat festgestellt, dafi man mit der und der Wassermenge eine normale Wurst berstellen kann; ich sehe nicht ein, warum man die Begrenzung nicht so treffen sollte - wenn ich ihn recht verstanden habe, schlägt er vor $20 \%$ - ich glaube also, für diese Ersatzwürste können wir rubig eine Grenze des Wasserzusatzes von $20 \%$ festlegen.

Prof. Krug: Die Ausführungen des Herrn Kollegen Behre bezw. die Erfahrungen, die er mit der Feder'schen Zahl für reines Fleisch gemacht hat, haben mich etwas überrascht, weil sie im Widerspruch stehen mit den Feder'schen Untersuchngen, wie auch mit unseren Ergebnissen. Wir konnten nämlich in keinem Fall eine Verhältniszahl nach Feder von uber 4 feststellen, obwohl bei der Untersuchung ein besonderer Wert darauf gelegt wurde, dak die Proben von Tieren mit verschiedenem Ernährungszustand und Alter, wie auch von verschiedenen Körperteilen entnommen waren. Die Richtigkeit der B e h r $\theta$ 'schen Untersuchungen vorausgesetzt, würde natürlich die Grandlage für die jetzige Beurteilungsnorm fallen und die Zahl 4 könnte nicht mehr als Grundlage in gerichtlichen Fallen herangezogen werden. Wir haben immer sehon aus praktischen Erwägungen, namentlich um den durch die Kriegsverhältnisse bervorgerufenen ungünstigen Verbältnissen nach Möglichkeit Rechnung zu tragen, bei einer Verhältniszehl yon wenig mehr als 4 von einer Beanstandung Umgang genommen und erst dann beanstandet, wenn die Zahl 5 übrschritten war. Ich glaube auch, dak man in diesem letzten Falle keinem Metzger ein Unrecht tut, denn der Konsument hat doch seblieflich auch ein Recht darauf, daf er gegen derartige stark mit Wasser beschwerte Wurstwaren geschiitzt wird, da erfahrungsmäfig solche Wurstwaren sehr bald verderben und aukerdem ganz wesentlich in ihrem Nährwerte herabgesetzt sind. Schlieflich ist es doch aurh unsere Pflicht darauf zu sehen, daß man die Leberwurst noch mit der Gabel essen kann und nicht genötigt ist, den Löffel $\mathrm{zu}$ verwenden, wie es jetzt leider vielfach der Fall ist.

Geheimrat Juckenack: Ich möchte nur ganz karz folgendes sagen: Die Frage des Wassergebaltes hat zurzeit eine ganz erhebliche Bedeutung, insbesondere aus den Gründen, die Kollege B ey thien angeführt hat: wegen der Ersatzwürste. Nun wäre die Sache sehr einfach, wonn die Ersatzmittelstellen sich schlechthin auf den Standpunkt stellen könnten: Fs ist unwirtschaftlich, denn es führt nur zu einer Verteuerung des Fleischmaterials, wenn man aus Fleiseh Wurst anfertigt, also es ist am besten, das Fleisch als solches in den-Verkehr zu bringen. Es fragt sich nur, ob das praktisch so leicht durchführbar ist. Ein anderer Gesichtspunkt, 
der vielleicht noch in Erwägung zu ziehen wäre, ist der: Wir gestatten die Herstellung von Ersatzwürsten nur aus bestimmten Teilen des Tieres ; es dürfen der ganze Rücken, die Hinter* keulen und Vorderviertel nicht zu W urst verarbeitet werden. Das wäre schon ein Gesichtspunkt. Der Gesichtspunkt, den Herr F e a d l er vorgetragen hat, hat auch manches für sich. Ich bin selbst stutzig geworden, als ich lirmittelungen dariuber anstellen ließ, ob sich überhaupt etwas derartiges praktisch durchführen läbt; da habe ich gesehen, wie tatsăchlich die Preise sind, and zwar nicht nur in Berlin und den Grofstädten, wo an sich schon sehr viel höhere Preise gezahlt werden müssen, sondern anch in den Indastriegebieten, wo das Bedürfnis besteht, fär Millionen von Mensehen etwas zu beschaffen. Wenn man sich die Sache überlegt, macht man die Beobachtung, dafs selbst draufen in der Provinz vielfach heute die Kaninchen je nach Gewicht mit sehr bohen Preisen bezahlt werden; Tiere, die sich zur Herstellung von Dauerwurst und Schlackwurst besonders eignen, also Tiere, die im Durchschnitt ungefähr 8-12 Pfand wiegen, also grofe schwere Kaninchen, die eine gute Dauerwurst geben, kosten aber beute schon draufen das Pfund Lebendgewicht $3 \mathrm{M}$; f für 2-2,50 M. bekommen Sie nur Tiere, die vielleicht $2^{1 / 2}$ Pfund wiegen, und man kann wohl sagen: von Tieren, die ein Lebendgewicht. von $2^{1 / 2}$ Pfund haben, können Sie das Fleisch nicht als solches stückweise verkaufen. Es fragt sich also, wie man sich $\mathrm{zu}$ den sehweren Tieren stellen soll. Wenn nun das Pfund rebendgewicht 3 M. kostet, so kommt das Schlachtgewicht schon auf 6 M., und wenn Sie nun noch rechnen, dak, wenn Sie die Ware in die Grokstadt hineinbekommen wollen, Sie noch böheré Preise anlegen müssen, dann kommt schon das Muskelfleisch einschlıeklich Knochen auf mindestens 6,50-7 M. Da fragt es sich doch, wie sich die Bevölkerung zu diesen Preisen stellen würde. Aus volkswirtschaftliehen Gründen kann es demnach zweckmäßjig sein, die Verarbeitung des ganzen Tieres anf Wurst zu gestatten, damit die Wurst wenigstens zu Preisen in den Verkehr kommt, die für die Masse noch erschwinglich sind. - Za der weiteren Frage, wieviel Wasserzusatz gestattet werden kann, und ob ohne Wasserzusatz Preise herauszuwirtschaften sind, die dem Publikum noch begreiflich erscheinen, bemerke ich, dak ich es verstehen kann, daß die Ersatzmittelstelle Groß-Berlin dazu übergegangen ist, den in Rede stehenden hohen Wassergehalt zu genehmigen. Allerdings wäre es mir lieber, wenn weit geringere Wasserzusätze gemacht werden wärden; amtlich habe ieh noch nicht Gelegenheit gehabt, zu der Frage Stellang nehmen zu müssen; sollte sich hierzu demnächst ein Anlaß bieten, so wird zu prüfen sein, welches Übel unter den gegenwärtigen Verbältnissen für die Allgemeinhelt das kleinere ist. Im Hinblick hierauf halte ich es auch für wichtig, daßs über die F'eder'sche Zahl eine Besprechung stattfindet, um eine Kontrolle der Genehmigungsbedingungen $\mathrm{zu}$ ermöglichen. Ich halte also auch im Hinblick hierauf die Ausführungen des Herrn Feder für auferordentlich wichtig. Wie sich demnächst in Friedenszeiten die Verhältnisse gestalten werden oder dürften, kann zur Zeit unerörtert bleiben.

Dr. Vollhase: Ich möchte noch darauf aufmerksam machen, daf die Beschränkung des Wasserzusatzes zur Wurst auch aus dem Grunde von großer Bedeutnng ist, weil die Wurst um so weniger haltbar ist, je mehr Wasser sie enthält. Ich habe in dieser Hinsicht in letzter Zeit Stellung zu nehmen gehabt, and zwar bezüglich der Wurstwaren, die im Auftrage der Landeszentralbebörde von einzelnen, besonders beauftragten Fabriken hergestellt werden, sowie auch bezïglich der in Mecklenburg hergestellten Ziegen- und Kaninchenfleischwürste. Die im Auftrage der Regierung hergestellter Würste werden von den Fabriken nach den einzelnen Orten innerhalb Mecklenburgs versandt und gelangen hier zur Verteilang. Eine übermäfige Wässerung der Wurst muß duher im Interesse der Haltbarkeit nubedingt vermieden werden. - Eine seit ungefähr 2 Jahren bestebende Kaninchenwurstfabrik stellte, als sie eröffnet wurde, eine gekochte Mettwurst mit einem Wassergehalt von $63,7 \%$ und einer Feder'schen Verbältniszahl von 2,4 her. Das war 1917 ! 1918 besafi die Wurst derselben Fabrik einen Wassergehalt von $76,9 \%$ und eine Feder'sche Verbältniszahl von 5,52. Sie hatte demnach einen Mindestwasserzusatz von $26,8 \mathrm{~g}$ zu 100 Teilen ungewässerter Fleischmasse exfahren. Die Folge hiervon war, daj die Wurst schon in mehr oder weniger verdorheném Zustande in die Hände der Verbrancher kam. Wir haben daher hiergegen energische Maß3regeln getroffen. Ich selbst habe dann später Gelegenheit gehabt, die Fabrik zu revidieren und die Herstellung der Wurstwaren zu verfolgen. Wahrend der Besichtigung habe ieh sowohl von den Wurstmassen als auch von den fertigen Würsten Probe entnommen. Auf Grund unserer Untersuchungen kann ieh bestätigen, daß3 der Wasserzusatz, den man in der fertigen Wurst findet, stets recht erheblich zuräckbleibt hinter der Wassermenge, die in der Wurstmasse enthalten ist, d. h. tatsächlich zugesetzt wurde. So fand sich in der Wurstmasse von Brühwürstchen ein Mindestwasserzusatz zu 100 Teilen ungewässerter Flleischmasse von $36 \mathrm{~g}$; in den feitigen Würstchen dagegen fanden sich nur $26,4 \mathrm{~g}$.

Dr. Murdfield: Jch habe in der Praxis feststellen können, daf die Feder'sche Zahl auch bei der Beurteilung von Kaninchen- und Ziegenwurst durchaus brauchbar ist, und kann bestätigen, was verschiedene meiner Herren Vorredner auch schon gedagt haben, daf man mit Hilfe der Verhältniszahl 4 weniger Wasser findet als tatsächlich zugesetzt ist. Meine 
Erfahrungen beruhen auf solchen Fällen aus der Praxis, in denen die Ersatzwürste unter Angabe der zugesetzten Wassermengen zur Prüfung vorgelegt wurden, bei der analytischen Nachpräfung ergab sich dann bei Anwendung der Fed er'schen Zahl 4 regelmäkig, dak etwas weniger Wasserzusatz gefunden wurde, als angegeben. Die Prüfung verlief also immer zu gumsten des Herstellers. Es ergibt sich daraus, daß die Feder'sche Zahl 4 für die Ersatzwürste durchaus anwendbar ist. In bezug auf die praktischen Ergebnisse unserer Erörterungen möchte ich bitten, daß wir doch zu irgend einer lintscheidung kommen. Es werden Ersatzwiirste in den Handel gebracht, die sehr schwankend sind in ibrem Gehalt an zugesetztem Wasser, einer stellt sie mit $20 \%$, der andere mit $40 \%$ und der dritte sogar mit $70 \%$ her; man mus aber doch irgend eine Norm haben, um beurtellen zu können, wie weit man da mit der Zulassung gehen soll. Wenn man unter Berücksichtigung der Ausführungen von Fend ler, und Juckenack nicht festlegen will, daf höchstens $20 \%$ zugesetzt werden dürfen, kaun man ja schlieflich einen etwas höheren Zusatz zulassen, aber zu irgend einer Normierung des znlassigen Wasserzusatzes müssen wir kommen, damit wenigstens eine gewisse Einheitlichkeit gewährleistet und den allen Grundsätzen der Nahrungsmittelbeurteilung hohnsprechenden jetzigen Zuständen ein Hinde berejtet wird.

Dr. Sprinkmeyer: Ioh bin anch-der Ansicht, dak wir uns hente nicht festlegen auf einen Wassergehalt für die Markenwurst, sondern dak wir nur feststellen, welche Wasserzusätze sind für die Wurstersatzwaren zulässig, also für Kaninchenwürste, Ziegenwurst, insbesondere auch für Fischwurst, die jetzt sehr viel bei uns an der Wasserkante hergestellt wird and die, wie Kollege Feder mir mitgeteilt hat, hauptsächlich nicht aus frischen Fischen hergestellt wird, sondern aus Stockfisch mit einem Zusatz von Kartoffelu und Graupen, und da ist der Wasserzusatz im allgemeinen recht hoch; natürlich, der Zusatz von Kartoffel und Graupen ist auch nicht zulässig.

Dr. Leonhard: Es ist eben bereits gesagt"worden, was ich sagen wollte: Wir müssen einen Unterschied machen zwischen Wurst, die allgemein gegen Abgabe von Marken hergestellt wird, und markenfreier Wurst. Ich bin der Ansicht, das für markenfreie Wurst eine Grenze festgesetzt werden soll, wie für die anderen Wirste.

H. Trillich: Ich möchte nur betonen, dafs ich es schon für zweckmä̈ig halte, wenn Wurstwaren hergestellt werden und nicht an Stelle dessen das Fleisch direkt gegeben wird. Wenn man nämlich sieht, wie man in den Metzgerläden betrogen wird, wenn man Fleisch kaufen will, und dafür Kuochen erhält, dann ist es nicht za verwundern, wenn der Käufer schlieblich die Wurstware doch vorzieht, denn da hat man doch wenigstens etwas sicheres. Dann möchte ich noch oins sagen: Auf dem Gebiete dieser Wurstwaren sind in Deutschland noch recht verschiedene Bräuche zu finden, sodaf man von einer einheitlichen Feststellung überhaupt gar nicht sprechen kann. leh wüfte wirklich vicht, wio z. B. die Münchener Leberwurst and die Braunschweiger Leberwarst bezüglich ihres Wassergehalts und überhaupt ihrer Zusammensetzung miteinander verglichen werden können; ich glaube, dafis viele von den Differenzen zwischen einzelnen Sachverständigen schlieblich darauf zurückzuführen sind, das die Herren im Grunde genommen von ganz verschiedenen Dingen reden, wenn sie auch die gleichen Namen nennen. Man kann deswegen unter gar keinen Umständen beispielsweise allgemein einen Wasserzusatz von $20 \%$ festsetzen, das ist ganz unmöglich. Da müßite man schon für jede einzelne Wurstsorte nach dem betreffenden Landessebrauch für die Herstellung einen besonderen Höchstwassergehalt berücksichtigen, - ich wüGte wirklich nicht, wie man das sonst machen wollte.

Dr. Luning: Die Frage des Wassergehaltes der Wurst hat für die Konsumenten anch insofern eine große Bedeutung, als die Menge Wurst, auf die der einzelne Konsument ein Anrecht hat, infolge der Rationierung durchaus beschränkt ist. Wenn man sagt, der Konsument erhalte infolge eines höheren Wasserznsatzes mehr Wurst, so trifft das nicht za; er erhält nicht mehr als eine ganz bestimmte Menge und nach meiner Meinung hat er ein Anrecht darauf, diese in Form vom normaler Wurst zu erhalten, nicht als Wurst mit erhöhtem Wassergehalt. Gibt man ihm solche, so handelt es sich nicht mehr um A u to suggestion, sondern um Fremdsuggestion, auf Deatseh um, Tüuschung". Ich babe bei Beratungen ubr $r$ die Fleischverteilung seitens der Stadt in meiner Heimat immer darauf hingewiesen, dab dem Konsumenten ein Anrecht auf eine bestimmte Menge Wleisch zusteht and somit auch auf eine bestimmte Menge Fleisch in Form von Wurst. Erhält er z. B. $250 \mathrm{~g}$ Wurst, so muf diese Menge $200 \mathrm{~g}$ Fleisch ohne Knochen entsprechen, d. h. der Wasserzusatz darf nicht mehr als $25 \%$ betragen.

Dr. Riechen: Herr Kollege Behre hat vorhin bemāngelt, dak noch zu wenig Untersuchungsmaterial vorliege, um ein zoverlässiges Urteil über dio Feder'sche Verhälniszahl zu gewinnen. Ich möchte anregen, daf, wenn weitere Untersuchungen auf diesem Gebiete gemacht werden, doch auch berücksichtigt wird, wie alt das Tier war, von welchem Körperteil das Fleisch entnommen ist und wie sonst im allgemeinen die Eruäbrungsverbältnisse waren sonst kommen wir nachher wieder dazu, das, wenn einmal eine höhere Zahl gefunden wird, 
die Sache gleich verallgemeinert wird. Finden wir aber dann einmal eine höhere Zahl, so haben wir doch wenigstens gleich die Unterlagen, und können uns ein besseres Bild von der Sache machen. Ich möchte also deswegen anregen, bei zukünftigen Untersuchungen dies mehr zu berücksichtigen, als es bislang geschehen ist.

Prof. Rupp: Tch möchte den Antrag stellen, dafá wir, wie es schon angeregt worden ist, die Feststellung über den Wassergehalt der Wurstwaren zurückstellen biṣ zum Frieden, während wir für die Ersatzwurstwaren einen Wassergehalt feststellen können, und zwar auf Grund der Feder'schen Verhätniszahl.

Dr. Seel: Ich möchte bemerken, dak wir anch das nicht machen können. Wir haben in den verschiedenen Gegenden Deutschlands die verschiedensten ortsüblichen Bräuche and Vorschriften für alle diese Sachen, und da läft sich eben keine Einheirlichkeit im Reiche erzielen. Auferdem stofen wir dabei auf Widerstände der Schlachthausdirektoren, mit denen wir unbedingt rechnen müssen, denn diese nehmen im allgemeinen einen gelinderen Standpunkt oin. Teh habe z. B. vor einigen Jahren eine Sache durchgefochten, bei der ich Beanstandungen machen mufte, weil es sich um den Gehalt an wirksamen Substanzen gehandelt hat; da wurde mir auch gesagt, wir sollten nicht so scharf sein, und jetzt in der Kriegszeit will man das selbstverständlich erst recht nicht haben. Ich glaube, wir dreschen über die ganze Wurstgeschichte leeres Stroh; es ist wirklich schade um die Zeit.

Dr. Le onhard: Für die Friedenswurst gibt es ortsübliche Herstellungsweisen, die in verschiedenen Gegenden verschieden sind, für die Kriegswurst gibt, es derartige ortsübliche Herstellungsweisen nicht; es haben sich da allerhand Miß̉bränche herausgebildet, die können aber jetzt, wo die Sache noch im Entstehen begriffen ist, noch erfolgreich bekämpft werden und kein Hersteller kann sich darauf berufen, er habe die Wurst nach ortsüblichem Brauch hergestellt - das gibts einfach nicht.

Dr. Fendler: Ich möchte darauf aufmerksam machen, daf meine Ausführungen sich nur auf Frischwurst bezogen. Von der Ersatzmittelstelle Groß̉-Berlin wird keine Dauerwurst genehmigt, weil zur Herstellung von Dauerwurst viel mehr Fleisch erforderlich ist, was nicht im Interesse der beabsichtigten Streckung des Kaninchen- und Ziegenfleisches liegt. Wenn Mettwurst, wie es in Schwerin geschehen ist, mit so hohem Wassergehalt hergestellt wird, so halte ich das fürr gänzlich unzulässig.

Dr. Murdfield: Aufer Brüh und Bratwürstchen handelt es sich meines Wissens bei den Ersatzwürsten vornehmlieh um sog. Kaiserjagdwurst, nach Art der Berliner Fleischwurst, mithin stets um Frischwürste, während eigentliche Dauerwürste aus Kaninchen- und Ziegenfleisch bisher wohl kaum hergestellt wurden (Zurufe: Doch). Es käme jedenfalls aber in erster Linio darauf an, daßs für die Frischwürste eine gewisse Norm für den zulässigen höchsten Wasserzusatz festgelegt würde. Zum Zwecke der Ausschaltung unfrachtbarer Erörterungen möchto ich nochmals darauf hinweisen, daf es sich heute nicht darum handelt, daf wir bestimmte Normen für die Beurteilung aller möglichen Wurstsorten im Sinne des Nahrungsmittelgesetzes aufstellen wollen, sondern daf wir gewisse Richtlinien haben wollen für die Benrteilung des zulässigen Wasserzusatzes bei der Prüfung und Beurteilung der Ersatzwürste im Sinne der Verordnungen, betreffend die Genehmigungspflicht von Ersatzlebensmitteln.

Dr. Cantzler: Es ist vorhin schon die Frage aufgeworfen worden, ob es nicht uberhaupt unwirtschaftlich sei, diese Ersatzwürste herstellen zu lassen. Ich kann mitteilen, dak wir in Mannheim auch Ziegenwürste gehabt haben; aber die Landespreisstelle hat die Herstellung einfach nicht genehmigt; die Folge war, daß das Ziegenfleiseh wesentlich billiger geworden ist; der Preis für Ziegenfleisch ist jetzt ich glaube auf 1,80 M. für das ganze Grof. herzogtum Baden festgesetzt worden. Ich halte die Verwendung dieses Fleisches für die Wurstzubereitung für unzweckmäfig; denn es wird dadurch nur der Preis für die geringe Menge Fleisch, das noch markenfrei verkauft werden kann, gewaltig in die Höhe getrieben. Wir haben in Mannheim kein Interesse daran, da bei uns Ziegenwurst nicht mehr hergestellt werden darf.

Prof. Behre: Es scheint doch so, als wenn der gröfte Teil der Anwesenden dafür zu haben ist, eine Grenze für den Wassergehalt bei Ersatzware (markenfreier Ware) festzusetzen, die auf Marken verteilte $W$ are aber aufer Betracht zu lassen. Das empfiehlt sich auch aus dem Grunde, den ich schon angeführt babe, nämlich weil von oben her ein Druck ausgeübt wird auf die Fleischerinnungen, and wir kein Recht und keine Möglichkeit haben, dem entgegenzuwirken. Ich glaube also, das Ergebnis unserer Aussprache lä̧̧t sich dahin zusammenfassen, daG wir für die Ersatzware einen bestimmten Wassergehalt für wünschenswert halten, vielleicht in Höhe von $20 \%$, daß wir den also als Grundlage für die neuen Beratungen über die Ersatzlebensmittel, die demnächst stattfinden sollen, empfehlen. Im übrigen ist diese ganze Frage des Wasserzusatzes bei den Ersatzwürsten in der Hauptsache eine Preisfrage. Diese Ersatzwürste sind ja genehmigungspflichtig, und es bleibt jeder Ersatzstelle nun vorbehalten, festzustellen, wieviel Wasser im einzelnen bei der Wurst zugelassen werden soll, das muß ja nachher im Preise zum Ausdruek kommen. Wenn wir aber hente sagen, wir wollen 
einen Zusatz bis 20\% zulassen, so ist das fur die Ersatzmittelstellen doch immerhin eino gewisse Grundlage, an die sio sich halten können.

Der Vorsitzende: Das Wort wird nicht mehr gewtinscht; wir kommen numeler zur Abstimmung über die Erage. Vorher möchte ich, um volle Klarheit zu schaffen, im Interesse der beiden Herren Referenten noch sagen, das es sich hier nicht um einen Beschluf handelt aber den Umfang, in welchem eine Wurstuntersvchung nötig, ist, um ein Urteil ïber ihre Gitte zu gewinnen, sondern es handelt sich hier nur darum, ob wir den Wassergehalt in einer fertigen Wurstware beschränken wollen oder nicht. Nun hat sich im Laufe der Debatte die Sache so zugespitzt, daf es von den meisten Rednern für richtig anerkannt wixd, daß wir für die jetzt hergestellte Kriegsware einen Wassergehalt vorschlagen, der nicht überschritten werden soll, und da hat, glaube ich, Herr Leonhard ganz richtig zum Ausdruck gebracht, dak wir das unbeschadet tun können, weil bei den Ersatzwinrsten von Gewohnheiten und Bräuchen nicht geredet werden kann. Für die Friedensware können wir einen Beschluf über den zulässigen Wassergehalt hente nicht fassen, sondern den wollen wir für spätere Zeit verschieben, weil heute diese Wurst nur eine ganz untergeordnete Rolle in der Ernâhrung spielt. Dagegen bin ich der Meinung, es wurde wundernehmen, wenn wir taber die Kriegsware nicht einen Beschluk fakten, oder doch fasten etwa in der Richtung, daf wir die Normierung eines Wassergehaltes nicht für nötig bielten. Ich halte es durchaus für nötig, weil in der Beziehung ganz zweifellos großer Unfug getrieben wird und viele Sachen vorkommendie man nicht dulden kann. Es kann sich jetzt nur darum hapdeln, die Höhe des Wassergehaltes festzusetzen (Zurufe: $20 \%$ ). Jawohl (Zuruf: Auf oder in Hundert). Wir müssen wohl auf Hundert sagen - also, ein Wassergehalt von $25 \%$. (Zuruf: Ist es nicht zweckmäfig, die Richtlinien des Herrn Feder noch einmal zur Verlesung zu bringen?) Herr Kollege Feder, wollen Sie noch einmal veriesen? (Verlesung.)

Dr. Willeke: Ich bitte den letzten Satz wegzulassen, wir würden uns sonst auf die Zahl 4 festlegen.

Dr. Feder: Ich habe für diesen Fall noch eine besondere Beanstandungsgrenze vorgeschlagen.

Dr. Willeke: Im ubrigen, glaube ich, können wir den Leitsatz annehmen, wir tun niemandem damit weh, weil wir mit der Zahl 4 regelmäßig weniger Wasserzusatz finden als tatsächlich zugesetzt ist.

Dr. Feder: Ich habe diese Zusätze nur vorgeschlagen als wirklichen Zusatz bei der Bereitung; dagegen habe ich als Beanstandungsgrenze einen anderen Gehalt vorgeschlagen, und zwar habe jch dies zum Ausdruck gebracht in folgendem Satze (Verlesung).

Der Vorsitzende: Ioh glaube, wir müssen uns etwas bestimmter ausdrücken, wenn unsere Beschlüsse als Grundlage dienen sollen.

Dr. Fendler: Ich möchte einen weitergehenden Antrag stellen. Ich beantrage, in die Richtlinien die Bestimmung aufzunehmen, dafs die Herstellung von Würsten aus Kaninchenand Ziegenfleisch untersagt wird, denn nur auf diese Weise wïrde es noch möglich sein, der Allgemeinheit markentreies Fleisch za erhalten. Wenn wir einen solch geringen Wassergehalt festsetzen, wie er vorgeschlagen worden ist, werden jedenfalls auf dem Berliner Markt für die Minderbegüterten die Ersatzwürste unersehwinglich. Ich möchte also den Antrag stellen, daß3 die Herstellung von Ziegen- und Kaninchenwürsten überbaupt untersagt wird. würde.

Der Vorsitzonde: Ich weib nicht, ob das unsere Zustundigkeit nicht übersehreiten

H. Trillich: Ich glaube, dieser Antrag zeigt, dak ich mit dem recht habe, was ich eben sagen wollte: Ob wir nicht überbaupt in der Frage zu weit gehen. Auch in der Frage des Wasserzusatzes - denken Sie nur daran, welche Mühe es schon kostet, bei der jetzigen Fleischnot der städtischen Bevölkerung anch nur diese kleinen Mengen Fleisch zuzuteilen; da ist es doch nun vielleicht immerhin eine Täuschung, aber doch eine angenehme Täuschung fín die Leute, wenn sie ein gröberes Stück Wurst dadurch bekommen, daf sie mit etwas mehr Wasser zubereitet ist - es sieht doeb immerhin nach etwas mehr aus. Ich bin überzeugt, wenn hente Vertreter der Reichsregierung and der Fleischversorgangsstelle bier wären, so wî̉rden sie dringend bitten, von allen derartigen Beschlüssen heute abzusehen, denn möglicherweise sind sie morgen schon nicht mehr durchführbar oder gerade das Gegenteil von dem, was zweckmäbig ist.

Der Vorsitze nde: Sie vergessen, daf das Ganze eine Preisfrage ist; der tabermäfige Wasserzusatz macht es den Herstellern solcher Würste möglich, sich übermäßig zu bereichern. Aus dem Grunde müssen wir doch einen Wasserzusatz normieren, der für die Berechnung des normalen Preises zugrunde zu legen ist.

Dr. Rothonfuker: Es ist, soviel ich sehe, klar, daf für die normalen Würste nichts zu machen ist, aber für die Ersatzwürste, für die eine Ortsäblichkeit bezüglich der Herstellung nicht besteht, sind wir nicht gebunden. Ich glaube, dafs wir auf das richtige kommen und 
beiden Teilen gerecht werden, wenn wir sagen: Für alle Ersatzwürste lassen wir anf 100 Teile Wurstmasse 20 Teile Wasser zu.

Reg.-Rat Dr. A u erbach: Ich wollte auch empfehlen, daß von den beiden Vorschlägen, die Herr $\mathrm{Feder}$ gemacht hat, derjenige zur Grundlage der Beschlüsse genommen wird, der sich auf die Herstellang bezieht, nicht aber auf die Untersuchung - es bleibt dem Chemiker unbenommen, ein anderes Untersuchungsverfahren zugrunde zu legen. Ich kann also nur das unterstützen. was Herr Rothe $\mathrm{f} u \dot{b} \mathrm{er}$ angeregt hat.

Der Vorsitzende: Ws ist also jetzt der Vorschlag gemacht worden, den ich auch unterstützen wollte, daf der Wasserzusatz bei Ersatzfleisehwürsten (Frischwürste) höchstens $20 \%$ betragen darf, d. h. anf 100 Teile Fleisch 20 Teile Wasser. (Zuruf: Ohne Anführung der einzelnen Sorten.) Ich halte es auch für sebr wertvoll, wenn nur eine einzige Zahl angefuhrt wird - also für alle Würste, Blutwürste wie Leberwürste -, sonst sind wieder neue Berechnungen notwendig.

Prof. W. Fresenius: Wenn wir sagen auf hundert Teile ungewässertes Fleiseh, ist da bei Blutwurst das Blut zum Fleische mitgerechnet oder nicht? Wenn nun darin gar kein Fleisch ist, so haben wir für Blutwurst gar keine Grenze (Zuruf: Kommt nicht in Frage!).

Reg.-Rat Dr. Auerbach: Es heibt: Auf 100 Teile ungewässerte "Wurstmasse", so war es formuliert.

Dr. Leonhard: Ich maf leider darauf hinweisen, daf anch bei dieser Fassung ein Irrtum möglich ist. Man darf nicht sagen: A af 100 Teile ursprünglicher Wurstmasse, sondern es muf heiken: Auf 100 Teile ursprüngliches Fleiseb, denn die Wurstmasse, die verwendet wird, kann gekocht sein, und damit kommt man zu ganz anderen Zahlen, weil beim Kochen ganz erhebliche Mengen Wasser herausgehen. Wenn man eine Beurteilung im Sinne des Herrn Feder überhaupt vornehmen will, mús man unbedingt zurückgreifen auf das ursprüngliche Fleisch, also auf 100 Teile ursprüngliches Fleiseh dürfen nicht mehr als 20 Teile Wasser zuge setzt werden.

Geheimrat Juckenack: Dann müssen Sie bei Fleisch in Klammern eine nähere Definition hinzusetzen.

Dr. Feder: Ich möchte diesem Vorschlage entgegenhalten, daß sich das tatsächlich gar nicht durchführen läßst. Wie soll man die Teile des urspriinglichen Fleisches wiegen? Bei dem Kleinvieh ist es ganz ausgeschlossen, die Kaninchen können nur vollständig ansgebeint werden, wenn sie ganz und gar weich gekocht sind. Dasselbe ist bei Rinderköpfen usw. der Fall. Das sind Schwierigkeiten, die es technisch unmöglich erscheinen lassen, die Teile ursprünglichen Fleisches zur Schätzung zu bringen.

Dr. Rothenfußer: Mein Antrag lautet auf Gebrauch des Ausdruckes: "Wurstmasse". Es ist nicht richtig "Fleisch" zu sagen, weil da die Schwierigkeiten entstehen, "auf die schon hingewiesen worden ist, es muß heißen: "Ungewässerte Wurstmasse".

Dr. Willeke: Bei Leberwurst ist $20 \%$ auch berechtigt, denn wenn die Leberwurst auch tatsächlich meist aus gekochtem Fleisch hergestellt wird, so wird sie doch meist angeränchert verkauft und der Verlust, der beim Räuchern entsteht, gleicht den Zusatz wieder aus.

Dr. Sprinkmeyer: Ich möchte noch eiumal die Frage anschneiden, wie es mit dem Wasserzusatz bei Fischwurst gehalten werden soll, die aus Stockfisch hergestellt ist, sie kommt bei uns sehr viel in den Handel.

Geheimrat Juckenack: Ich glaube, daß man sich da ohne weiteres auf den Standpunkt stellen müßste, daßß durch die Verarbeitung von Stockfisch auf Wurst der Stockfisch in einer Weise vertenert wird, die mit den Grundsätzen der Verordnung nicht im Einklange steht.

Der Vorsitzende: Herr Leonhard, würden Sie Ihren Antrag zurickziehen?

Dr. Leonhard: Ich verzichte auf das Wort. Es liegt ein Mikverständnis vor, das, um die Verhandlangen nicht zu lange hinauszuziehen, hier nicht aufgeklärt werden kann.

Dr. Fendler: Ich vermag nicht einzusehen, warum wir nicbt in dex Lage sein sollen, vorzuschlagen, dal' Ersatzw ürste aus Kaninchen* and Ziegenfleisch nicht hergestellt werden dürfen. Ich glaube nicht, daßs wir damit aber unsere Kompetenzen hinausgehen würden.

Der Vorsitzende: Als ich gesagt habe, es besteht das Bedenken, ob das nicht unsere Zuständigkeit überschreiten würde, bin ich dabei von der Erwägung ausvegangen, daß wir uns hier in dieser Versammlng im wesentlichen mit der Untersuchung und Beurteilung von Nahrungsmitteln beschäftigen und nicht damit, ob es zweckmäßig sei, irgend ein Nahrungsmittel herzustellen oder za verbieten. Nur von dem Gesichtspunkte aus habe ich geurteilt, sonst wïrde ich mich als Mensch für genau so kompetent, balten wie die Preisprüfungsstellen, die daruber zu befinden haben. A ber es scheint eine allgemeine Stimmung dagegen zu sein. (Widerspruch.) Wir würden dann auf den Antrag zurückkommen, das wir sagen: dak auf 100 Teile ungewässerter Wurstmasse nicht mehr als 20 Teile Wasser zugesetzt sein sollen, und zwar gilt das für alle Wurstarten (Zuruf: Ersatzkriegswiurste). Bei der Berechnung haben wir uns auf die Feder'sche Zahl nicht festgelegt. Das muf der Zukunft vorbehalten bleiben, wenn wir erst wieder normale Friedensware zu unseren Untersuchungen bekommen. 
Wir haben uns im Sinne der Ausführungen des Herm Seel daräber ausgelassen, in welchem Umfange überhaupt eine Beurteilung von Wurstwaren notwendig ist. Und selbstverständlich gehört dazu auch die mikroskopisehe Untersuchung.

Der Vorsitzende: Damit können wir diesen Punkt verlassen und kämen zum folgenden Punkte: „Brühwürfel und Suppen in trockener Form". Zunächst möchte ich die Herren fragen, ob Sie gewillt sind, jetzt noch weiter zu tagen (Rufe: Nein!). Dann möchte ich bitten, morgen punkt 9 Uhr wieder hier zu sein. (Zuruf 1/29 Uhr - Widerspruch.) Ich nehme an, daß unsere geschäftlichen Verhandlungen mindestens 2 Stunden in Anspruch nehmen werden. Ich teile zu Ihrer Orientierung mit, dab die neuen Satzungen, die wir beraten wollen, morgen nur in erster Lesung besprochen werden; die zweite Lesung wird erst übers Jahr stattfinden, sodaß wir mit Beratung der Satzungen und auch mit der Beratung der Standesinteressen wohl in 2 Stunden fertig werden und um 11 Uhr unsere öffentliche Versammlung wieder beginnen können, und zwar werden wir wieder einsetzen mit unserer Tagesordnung unter Ziffer $3^{b}$, wir würden also zunächst über Brühwürfel und Suppen in trockener Form zu sprechen haben.

(Schluß der 1. Sitzung abends 6 Uhr.)

\section{Sitzung, Samstag, den 28. September 1918.}

Den Beginn der 2. Sitzung bildeten Beratungen über $\mathrm{Standesfragen} \mathrm{und} \mathrm{einen}$ Entwurf neuer Satzungen des Vereins, worüber gesondert berichtet werden wird.

Der Vorsitzende: Wir kommen dann zu unserer wissenschaftlichen Tagesordnung und haben nunmehr in der Reihenfolge zunächst fortzufahren unter Ziffer $3^{b}$ Brühwürfel, Suppen in trockener Form. Ich habe dann die, Absicht, nachdem Punkt $3^{b}$ erledigt sein wird, da unsere Zeit sehr knapp ist, zunächst nicht den Punkt 5, sondern erst den Punkt 6 zur Erledigung zu bringen: „Hat es sich als wünschenswert erwiesen, für die Untersuchung von Ersatzlebensmitteln bestimmte Verfahren vorzuschreiben? - weil dafür ein sehr großes Interesse vorhanden ist. Vorher hat das Wort für eine Mitteilung Herr Murtfeldt.

Dr. Murtfeldt: Vor einiger Zeit wurde uns ein Brot aus einer Mindener Bäckerei von M. und drei halbe Brote aus der Verkaufs-Nebenstelle von F. zur Untersuchung und Begutachtung überbracht. Alle vier Brote zeigten auf den Schnittflächen starke Rasen eines orangefarbenen Schimmelpilzes (Oidium aurantiacum). Nach dem Genuß des Brotes waren Verdauungsstörungen eingetreten. Drei von diesen Broten waren nach dem Ankauf 8 Tage in einer Speisekammer aufbewahrt worden, bei dem Anschneiden hatten sie gedampft, und einen Tag nach dem Anschneiden zeigten sie orangefarbene Stellen. Bei der Einlieferung ins Untersuchungsamt waren die Brote noch warm; ein in das Brot eingeführtes Thermometer hielt sich 10 Tage hindurch auf $42^{\circ}$ (Temperatur im Aufbewahrungsraum 18-20 ). Die Schnittflächen der Brote und Stellen unter der gelockerten Rinde bedeckten sich in kurzer Zeit mit weit ausgebreiteten, orangefarbenen Pilzrasen, im Innern hatte die Krume eine weiflich-gelbe Marmorierung angenommen. Durch das Oidium aurantiacum ist eine völlige Zersetzung der Kohlenhydrate unter starker Entwickelung von Kohlensäure erfolgt. Die Zersetzung ging bis zur Bildung von Alkohol weiter. Nach $\mathrm{K}_{0} \mathrm{nig}^{\mathbf{1}}$ ) ist die Bildung von Alkohol bisher nur bei Aspergillus nidulans beobachtet worden.

1) J. König, Chemie der menschlichen Nahrungs- und Genufmittel. IV. Aufl., Berlin $1904,2,681$. 
Ich erlaube mir, die Photographien Ihnen vorzulegen. Herr Prof. Dr. Bömer, dem ich die Photogramme einsandte, hatte Bedenken, die farbigen Photographien in die Zeitschrift aufnehmen zu lassen, weil das Schwierigkeiten bot, und die nichtfarbigen Photographien zu wenig instruktio wären.

\title{
Fleischbrühersatzwürfel.
}

\author{
Von \\ Dr. R. Murdfield-Hamburg.
}

Durch die Bundesratsverordnung über Fleischbrühwürfel und deren Ersatzmittel vom 25. Oktober 1917') sind die ärgsten Mißstände im Handel und Verkehr mit diesen Erzeugnissen behoben worden. Die eigentlichen "Fleischbrühwürfel“, deren Beschaffenheit in erster Linie durch diese Verordnung (\$ 1) geregelt wurde, sind inzwischen wegen des immer mehr ansteigenden Mangels an Fleischextrakt so gut wie völlig vom Markte verschwunden. Eine um so stärkere Ausdehnung hat der Verkehr mit Ersatzmitteln hierfür genommen, deren Zusammensetzung im $\S 2$ der Verordnung gleichfalls eine gewisse Regelung erfahren hat.

In der laufenden Kontrolle der Fleischbrühersatzwürfel, sowie namentlich gelegentlich der Prüfung solcher Erzeugnisse nach Maßgabe der Bundesratsverordnung über die Genehmigung von Ersatzlebensmitteln vom 7. März $1918^{2}$ ) hat sich indessen ergeben, daß die Bestimmungen der Bundesratsverordnung vom 25. Oktober 1917 in bezug auf die Zusammensetzung der Ersatzwürfel noch nicht völlig ausreichen, oder der Lebensmittelüberwachung noch nicht in allen Fällen hinreichende Handhaben geben, um ungeeignete und minderwertige Erzeugnisse dieser Art ohne weiteres auszuschalten.

Insbesondere fehlt bei den Ersatzwürfeln ein analytischer Wertmesser für den Genuh- und Würzwert dieser Erzeugnisse, wie er für die eigentlichen Fleischbrühwürfel durch die Festlegung des Mindestgehaltes an Stickstoff (aIs Bestandteil der den Genußwert bedingenden Stoffe) und an Gesamt-Kreatinin im $\$ 1$ unter Ziffer 2 gegeben ist. Zwar wird auch für die Ersatzwürfel im $\$ 2$ ein gewisser Mindestgehalt an Stickstoff (als Bestandteil der den Genußwert bedingenden Stoffe) "gefordert, doch genügt diese Anforderung allein nicht, um einen hinreichenden Würz- und Genußwert dieser Erzeugnisse zu gewährleisten.

Die Fleischbrühersatzwürfel bestehen zumeist aus Kochsalz, Würzen oder sog. Extrakten (vornehmlich Knochenbrühextrakt), Auszügen aus Suppenkräutern und -gemüsen sowie Gewürzen. Der Kochsalzgehalt ist mit höchstens $70 \%$ vorgeschrieben; in bezug auf die übrigen Stoffe besteht aber nach Menge, Art und Wertbeschaffenheit weiter Spielraum, da in der Bundesratsverordnung nur ein Mindestgehalt an Stickstoff (als Bestandteil der den Genufwert bedingenden Stoffe) von 2\% vorgeschrieben ist. Nun sind aber nach unseren Erfahrungen die zur Herstellung der Ersatzbrühwürfel verwendeten Würzen, Extrakte $u$. dergl., durch welche in erster Linie die Fleischbrübähnlichkeit dieser Erzeugnisse bewirkt werden soll, nach ihrem Genuß- und Würzwert sehr verschieden. Diese Verschiedenheit kommt im Gehalte dieser Stoffe an Gesamt-

1) Reichs-Gesetzbl. 1917, 969; Gesetze und Verordnungen, betr. Nahrungs- und Genuf mittel, 1918, 10, 10.

2) Reichs-Gesetzbl. 1918, $113 \mathrm{ff}$. 